\title{
Homoclinic Saddle-Node Bifurcations and Subshifts in a Three-Dimensional Flow
}

\author{
Geertje Hek $^{1}$, Arjen Doelman ${ }^{1}$, Philip Holmes ${ }^{2}$ \\ ${ }^{1}$ Mathematisch Instituut, Universiteit Utrecht, P.O. Box 80.010, \\ 3508 TA Utrecht, the Netherlands. \\ ${ }^{2}$ Program in Applied and Computational Mathematics and \\ Department of Mechanical and Aerospace Engineering, \\ Princeton University, Princeton, NJ 08544, U.S.A.
}

April 22, 1998

\begin{abstract}
We study a two-parameter family of three-dimensional vector fields that are small perturbations of an integrable system possessing a line $\Gamma$ of degenerate saddle points connected by a manifold of homoclinic loops. Under perturbation, this manifold splits and undergoes a quadratic homoclinic tangency. Perturbation methods followed by geometrical analysis reveal the presence of countably-infinite sets of homoclinic orbits to $\Gamma$ and a non-wandering set topologically conjugate to a shift on two symbols (a Smale horseshoe). We use the symbolic description to identify and partially order bifurcation sequences in which the homoclinic orbits appear, and we formally derive an explicit two-dimensional Poincaré return map to further illustrate our results. The problem was motivated by the search for traveling 'structures' such as fronts and domain walls in partial differential equations.
\end{abstract}

\section{Introduction}

In this paper we continue the study of homoclinic and heteroclinic orbits in near-integrable three-dimensional flows begun in [3] and continued in [2]. The underlying idea is to study the global consequences of local (saddle-node) or quasi-local (homoclinic) bifurcations, whose presence and type can be determined by straightforward normal form or Melnikovtype calculations. Geometrical arguments involving a Poincaré map then build on the perturbation analyses to create a relatively complete global picture of stable and unstable manifolds and their intersection sets. In this manner we detect countable [3] or large [2] sequences of homoclinic and heteroclinic orbits and (partially) describe the bifurcations in which they are born.

The vector fields studied in $[3,2]$ are simple polynomials which represent key features of rational functions obtained by inserting a traveling wave Ansatz in PDEs of GinzburgLandau type, and the homoclinic or heteroclinic orbits correspond to spatial structures with one or more pulses or bumps (domains) or kinks (interfaces); see [1, 14] and references 
given therein. These structures are generally regarded as relevant to the larger problem of pattern-formation and spatio-temporal dynamics in extended systems.

The systems of concern here are of the general form

$$
\left\{\begin{aligned}
\dot{x} & =y \\
\dot{y} & =x-x^{2}+\varepsilon f(x, y, z ; \mu) \\
\dot{z} & =\varepsilon g(x, y, z ; \mu)
\end{aligned}\right.
$$

so that, for $\varepsilon=0$, the functions

$$
k(x, y)=\frac{1}{2} y^{2}-\frac{1}{2} x^{2}+\frac{1}{3} x^{3} \quad \text { and } \quad z
$$

remain constant and the lines $\Gamma_{0}=\{(x, y, z) \mid x=y=0\}$ (resp. $V_{0}=\{(x, y, z) \mid x=1, y=$ $0\})$ are filled with hyperbolic (resp. elliptic) equilibria. Each point on $\Gamma_{0}$ is connected to itself by a homoclinic loop: the compact part of the level set $k(x, y)=0$. For simplicity the functions $f$ and $g$, which depend upon a parameter $\mu \in \mathbf{R}^{d}$, are chosen to preserve these lines, but since $\Gamma_{0}$ is normally hyperbolic, a nearby slow manifold $\Gamma_{\varepsilon}=\Gamma_{0}+O(\varepsilon)$ is preserved for any $C^{2}$-small perturbations $f, g$ [4]. Our main goal is to study the stable and unstable manifolds $W^{\mathrm{s}}\left(\Gamma_{\varepsilon}\right), W^{\mathrm{u}}\left(\Gamma_{\varepsilon}\right)$ of $\Gamma_{\varepsilon}$ and their intersections: the homoclinic and heteroclinic orbits referred to above.

In [3] we took the quadratic functions $f=y(b+c z), g=\nu+\rho x+z^{2}$, for which a saddle-node bifurcation of fixed points $S^{ \pm}=\{(x, y, z)=(0,0, \pm \sqrt{\nu})\}$ occurs on $\Gamma_{\varepsilon}$, and the stable and unstable manifolds of $\Gamma_{\varepsilon}$ intersect transversely in a primary single pulse loop $\gamma(t)$ near $z=-\frac{b}{c}$. The focus of [3] is the interaction of $S^{ \pm}$and their stable and unstable manifolds with $\gamma(t)$, which leads to the creation (destruction) in explosions (implosions) of countable sets of secondary homoclinic or heteroclinic orbits connecting $S^{+}$to $S^{-}, S^{+}$ to $z=+\infty, z=-\infty$ to $S^{-}$and $z=-\infty$ to $z=+\infty$.

In [2] we took the ostensibly simpler functions $f=y\left(z^{2}-a\right), g=1+b x$ for which $W^{\mathrm{u}}\left(\Gamma_{\varepsilon}\right)$ and $W^{\mathrm{s}}\left(\Gamma_{\varepsilon}\right)$ have quadratic contact near $z=0$ at $a=a^{*}=0+O(\varepsilon)$. There are no fixed points on $\Gamma_{\varepsilon}$ in this case (although in [2] and below we sometimes find it convenient to compactify by introducing fixed points $S^{ \pm}$far from $z=0$ but with $\left.\left|z^{ \pm}\right|<\infty\right)$. We showed that the primary homoclinic tangency, which creates two singlepulse homoclinic orbits, is followed by a cascade of bifurcations creating $n$-pulse orbits $(n=2,3, \ldots, O(|\log \varepsilon|))$ as $a$ increases through $a^{*}$ to $a=O(1)$. If $b \geq-1$, these are the only such connections, but if $b<-1$ the picture is considerably more complex. In this case, the vertical component $\dot{z}=\varepsilon(1+b x)$ of the flow is positive for $x<-\frac{1}{b}$ and negative for $x>-\frac{1}{b}$. The existence of recurrent orbits which "balance" these counteracting effects can lead to the creation of a rich non-wandering set $\Lambda$ topologically conjugate to a shift on two symbols: a Smale horseshoe. In the present paper we prove the existence of such a set under appropriate assumptions on the parameters $(a, b)$, describe its symbolic dynamics, and use them to develop a symbolic description of orbits in $W^{\mathrm{u}}\left(\Gamma_{\varepsilon}\right) \cap W^{\mathrm{s}}\left(\Gamma_{\varepsilon}\right)$ and to describe the relationship between this latter homoclinic set, $\Lambda$ and $W^{\mathrm{u}}(\Lambda), W^{\mathrm{s}}(\Lambda)$. In doing so we extend and improve the bifurcation results in Section 6 of [2].

Section 2 reviews and extends the asymptotic calculations of [2], developing the necessary results on which the geometrical and symbolic analyses of Sections 3 and 4 are built. 
In those sections we construct the set $\Lambda$, recall its (semi-)conjugacy to the shift on two symbols, develop an analogous symbolic description to characterize homoclinic orbits in $W^{\mathrm{u}}\left(\Gamma_{\varepsilon}\right) \cap W^{\mathrm{s}}\left(\Gamma_{\varepsilon}\right)$, and describe a partial ordering of bifurcations in which these orbits are created. In Section 5 we formally derive an explicit two-dimensional mapping $F$ which models $\mathcal{P}$, show that it has a topological horseshoe for small $\varepsilon$ and yields explicit expressions for some of the bifurcation sets described earlier, and prove that it has a hyperbolic horseshoe for certain other parameter ranges. Section 6 contains conclusions.

Early papers in which such solutions to ODE boundary value problems of dimension $\geq 3$ were studied are those of Kopell and Howard [10, 12]. There the ODEs were derived from reaction-diffusion equations, and in the second paper horseshoes and spatially chaotic patterns were proven by the same techniques used in Sections 3 and 5 of the present paper (see also Section 6). This paper proceeds in a similar spirit, the goal being to provide a global understanding of a complex dynamical system and thereby to elucidate pattern formation.

\section{Review and extension of former results}

In this section we recall and extend the results of [2] needed for our subsequent analysis.

\subsection{Basic features of the model system}

We consider the system (1.1) with the functions $f$ and $g$ of [2]:

$$
\left\{\begin{array}{l}
\dot{x}=y \\
\dot{y}=x-x^{2}+\varepsilon y\left(z^{2}-a\right) \\
\dot{z}=\varepsilon(1+b x) .
\end{array}\right.
$$

Some analyses will require a compact normally hyperbolic slow manifold $\Gamma$ or a compactified flow in z-direction. Without changing the essential ingredients of the model system, these requirements can be met by putting two saddles far from $z=0$ on $\Gamma$ :

$$
\dot{z}=\varepsilon\left(1+b x-c z^{2}\right)
$$

with $1 \gg c \gg \varepsilon$, or even by placing invariant planes far from $z=0$ :

$$
\dot{z}=\varepsilon(1+b x)\left(1-c z^{2}\right),
$$

again with $1 \gg c \gg \varepsilon$. Replacing the third component of (2.1) by (2.2) or (2.3) affects neither the conclusions of the Melnikov calculations in this subsection, nor the calculations below in $\$ 2.2$, as long as $z^{2} \ll \frac{1}{c}$. (This $z$ range is sufficient, since the bifurcations we study occur in an $O(1)$ neighborhood of the $\{z=0\}$ plane).

Let $\Gamma=\Gamma_{\varepsilon}=\{x=y=0\}$. For $\varepsilon=0$ there exists a homoclinic manifold $\mathcal{H}$, corresponding to $k=0(1.2)$, which connects $\Gamma$ to itself. For $\varepsilon>0, \mathcal{H}$ splits into stable (resp. unstable) manifolds $W^{\mathrm{s}}(\Gamma)$ (resp. $W^{\mathrm{u}}(\Gamma)$ ), compact pieces of which contain orbits $O(\varepsilon)$ close to $\mathcal{H}$ for $t \geq 0$ (resp. $t \leq 0$ ) [4]. We study $W^{\mathrm{u}}(\Gamma)$ and $W^{\mathrm{s}}(\Gamma)$ via their intersections with the Poincaré cross section $\mathcal{V}=\left\{y=0,1<x<\frac{3}{2}+O(\varepsilon)\right\}$. We 
denote the "first" intersections of $W^{\mathrm{u}}(\Gamma)$ and $W^{\mathrm{s}}(\Gamma)$ with $\{y=0\}$, lying $O(\varepsilon)$ close to the component $\left\{y=0, x=\frac{3}{2}\right\}$ of $\mathcal{H} \cap\{y=0\}$, by $P(\Gamma)$ respectively $P^{-1}(\Gamma) . P$ is (formally) "half" the Poincaré return map $\mathcal{P}$ defined in Section 2.2 below. See [3] and Figure 1.

The Melnikov method for slowly varying systems gives an expression approximating the distance between $P(\Gamma)$ and $P^{-1}(\Gamma)$ as a function of $z$. Let $x_{\varepsilon}^{\mathrm{u}}$ and $x_{\varepsilon}^{\mathrm{s}}$ denote the intersections of $P(\Gamma)$ (resp. $\left.P^{-1}(\Gamma)\right)$ with $\left\{z=z_{0}\right\}$. Solutions $\gamma_{\varepsilon}^{\mathrm{u}}=\left(x_{\varepsilon}^{\mathrm{u}}, y_{\varepsilon}^{\mathrm{u}}, z_{\varepsilon}^{\mathrm{u}}\right)$ in $W^{\mathrm{u}}(\Gamma)$ and $\gamma_{\varepsilon}^{\mathrm{s}}=\left(x_{\varepsilon}^{\mathrm{s}}, y_{\varepsilon}^{\mathrm{s}}, z_{\varepsilon}^{\mathrm{s}}\right)$ in $W^{\mathrm{s}}(\Gamma)$ of equations $(2.1)$ are determined by the initial condition $\gamma_{\varepsilon}^{\mathrm{u}, \mathrm{s}}(0)=\left(x_{\varepsilon}^{\mathrm{u}, \mathrm{s}}, 0, z_{0}\right) ; \gamma_{0}(t)=\left(x_{0}(t), y_{0}(t), z_{0}\right)$ is defined as the homoclinic solution to the unperturbed system $(\varepsilon=0)$ with $\gamma_{0}(0)=\left(\frac{3}{2}, 0, z_{0}\right)$. Using the expression for the Melnikov function derived (for instance) in [16] we find that

$$
\triangle W\left(0, z_{0}, a\right)=\int_{-\infty}^{\infty} y_{0}^{2}(t)\left(z_{0}^{2}-a\right) d t=\frac{6}{5}\left(z_{0}^{2}-a\right) .
$$

Hence $\triangle W(0, z, a)=0$ for $z= \pm \sqrt{a}(a>0)$, which implies that for $\varepsilon>0, a>0, W^{\mathrm{u}}(\Gamma)$ and $W^{\mathrm{s}}(\Gamma)$ intersect transversely $O(\varepsilon)$ close to the planes $\{z= \pm \sqrt{a}\}$. Let $z_{ \pm}^{*}=\sqrt{a} \pm O(\varepsilon)$ denote the exact $z$ values for which $P(\Gamma)$ and $P^{-1}(\Gamma)$ intersect. Since $\triangle W(0,0,0)=$ $\frac{\partial}{\partial z} \triangle W(0,0,0)=0$ and $\frac{\partial^{2}}{\partial z^{2}} \triangle W(0,0,0) \neq 0, \frac{\partial}{\partial a} \triangle W(0,0,0) \neq 0$, a unique value $a=a^{*}=0+O(\varepsilon)$ exists, for which $W^{\mathrm{u}}(\Gamma)$ and $W^{\mathrm{s}}(\Gamma)$ have quadratic contact (cf. Theorem 4.5.4 of [6]). Let $z^{*}=0+O(\varepsilon)$ be such that for $a=a^{*} P(\Gamma)$ and $P^{-1}(\Gamma)$ are tangent at a point with $z$ coordinate $z^{*}$. The two intersections $W^{\mathrm{u}}(\Gamma) \cap W^{\mathrm{s}}(\Gamma)$ found for $a>a^{*}$ correspond to two single-loop homoclinic orbits $\gamma^{+}(t)$ and $\gamma^{-}(t)$ to $\Gamma$. These are defined such that $\gamma^{ \pm}(0)=\left(x_{ \pm}, 0, z_{ \pm}^{*}\right) \in P(\Gamma) \cap P^{-1}(\Gamma)$, where $x_{ \pm}=\frac{3}{2}+O(\varepsilon)$.

\subsection{Structure of $W^{\mathrm{u}}(\Gamma)$ and $W^{\mathrm{s}}(\Gamma)$ for different parameter values}

We approximate the Poincaré map on the cross section $\mathcal{V}=\left\{y=0,1<x \leq \frac{3}{2}+O(\varepsilon)\right\}$ to investigate the flow inside the manifolds $W^{\mathrm{s}}(\Gamma)$ and $W^{\mathrm{u}}(\Gamma)$. Define

$$
\mathcal{P}(k, z)=(k+\triangle K(k, z), z+\triangle Z(k, z))
$$

with $k \in\left(-\frac{1}{6}, 0\right)$ as in $(1.2)$. Repeated application of $\mathcal{P}$ to $P(\Gamma)$ and $P^{-1}(\Gamma)$ will yield further intersections of $W^{\mathrm{u}}(\Gamma)$ and $W^{\mathrm{s}}(\Gamma)$ with $\mathcal{V}$, leading to insight on the structure of these manifolds. For $n>1$ we define:

$$
\begin{array}{ll}
P^{n}(\Gamma) & \equiv \mathcal{P}^{n-1}(P(\Gamma)), \\
P^{-n}(\Gamma) & \equiv \mathcal{P}^{-n+1}\left(P^{-1}(\Gamma)\right) .
\end{array}
$$

A solution $\left(x_{\varepsilon}(t), y_{\varepsilon}(t), z_{\varepsilon}(t)\right)$, with initial data on $\mathcal{V}$, returns to $\mathcal{V}$ after a time $T_{\varepsilon}(k, z)$. The quantities $\triangle K(k, z)$ and $\triangle Z(k, z)$ measure the accumulated change in the variables $k$ and $z$ within this time interval. Thus, taking the compactified case (2.2), we have

$$
\begin{aligned}
\triangle K(k, z) & =\int_{0}^{T_{\varepsilon}(k, z)} \dot{k}\left(x_{\varepsilon}, y_{\varepsilon}, z_{\varepsilon}\right) d t=\varepsilon \int_{0}^{T_{\varepsilon}(k, z)} y_{\varepsilon}^{2}\left(z_{\varepsilon}^{2}-a\right) d t \\
\triangle Z(k, z) & =\int_{0}^{T_{\varepsilon}(k, z)} \dot{z}\left(x_{\varepsilon}, y_{\varepsilon}, z_{\varepsilon}\right) d t=\varepsilon \int_{0}^{T_{\varepsilon}(k, z)}\left(1+b x_{\varepsilon}-c z_{\varepsilon}^{2}\right) d t
\end{aligned}
$$




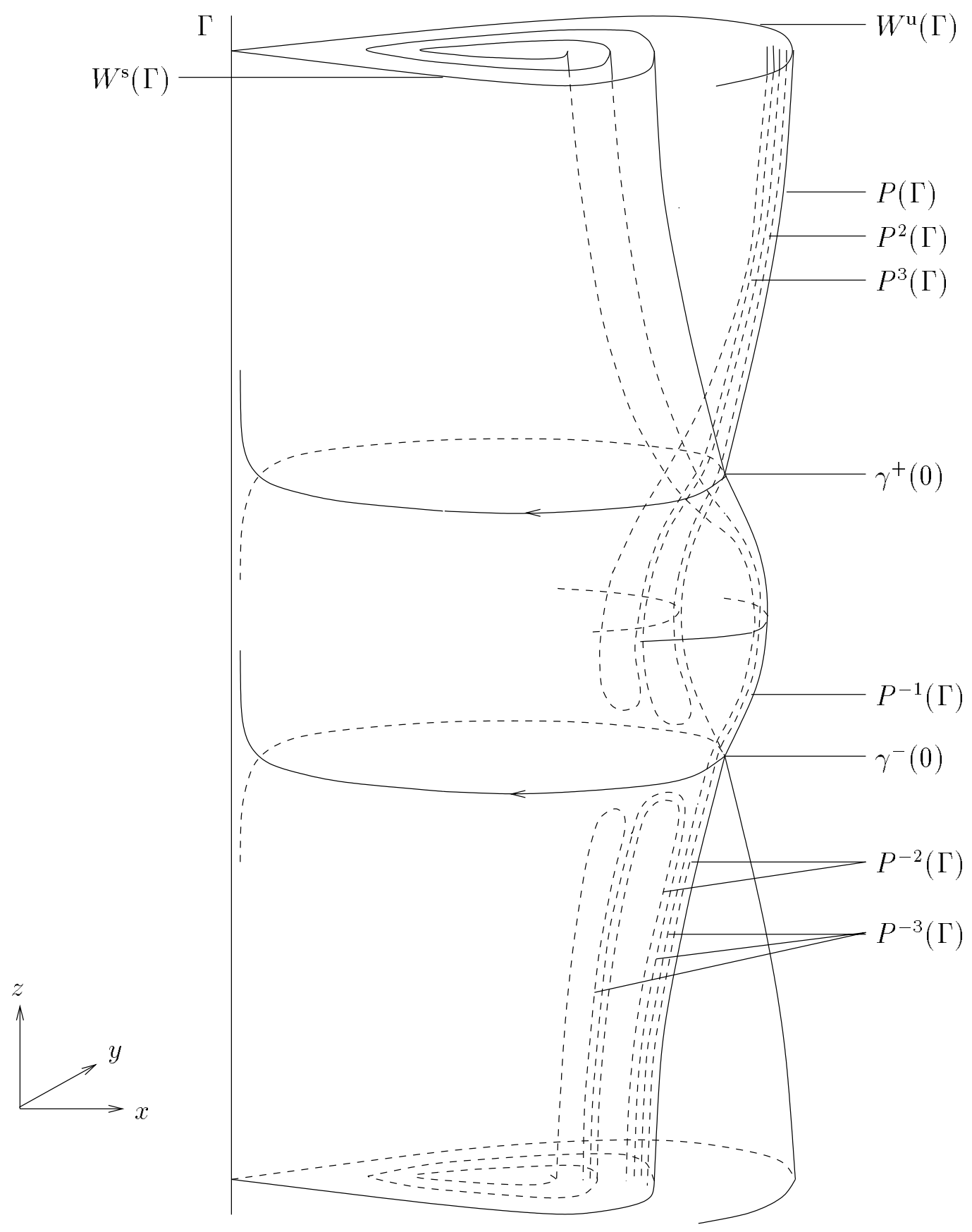

Figure 1: The structure of $W^{\mathrm{s}}(\Gamma)$ and $W^{\mathrm{u}}(\Gamma)$ for $a>0, b \geq-1, a, b=O(1)$. 
(For (2.1), simply set $c=0$.) We approximate $T_{\varepsilon}$ and $\left(x_{\varepsilon}(t), y_{\varepsilon}(t), z_{\varepsilon}(t)\right)$ by $T_{0}$ and $\left(x_{0}(t), y_{0}(t), z_{0}(t)\right)$ : the solution of $(2.1)$ with $\varepsilon=0$ and period $T_{0}$ and the same or $O(\varepsilon)$ close initial data. This yields

$$
\begin{aligned}
& \triangle K\left(k, z_{0}\right)=\varepsilon \int_{0}^{T_{0}\left(k, z_{0}\right)} y_{0}(t)^{2}\left(z_{0}^{2}-a\right) d t+O\left(\varepsilon^{2}\right), \\
& \triangle Z\left(k, z_{0}\right)=\varepsilon \int_{0}^{T_{0}\left(k, z_{0}\right)}\left(1+b x_{0}(t)-c z_{0}^{2}\right) d t+O\left(\varepsilon^{2}\right) .
\end{aligned}
$$

or, after changing variables,

$$
\begin{aligned}
& \triangle K(k, z)=2 \varepsilon \int_{x_{1}(k)}^{x_{2}(k)}\left(z^{2}-a\right) \sqrt{2 k+x^{2}-\frac{2}{3} x^{3}} d x+O\left(\varepsilon^{2}\right), \\
& \triangle Z(k, z)=2 \varepsilon \int_{x_{1}(k)}^{x_{2}(k)} \frac{1+b x-c z^{2}}{\sqrt{2 k+x^{2}-\frac{2}{3} x^{3}}} d x+O\left(\varepsilon^{2}\right) .
\end{aligned}
$$

Here $0<x_{1}(k)<1<x_{2}(k)<\frac{3}{2}$ are the points where the unperturbed orbit intersects the plane $\{y=0\}$.

We can now distinguish two cases. If the averaged flow in the fast field is in the same direction as the flow on $\Gamma(\triangle Z(k, z)>0)$, the system is called cooperating; if the averaged flow in the fast field is opposed $(\triangle Z(k, z)<0)$, the system is counteracting. To determine the different regions in parameter and phase space we estimate $\triangle Z(k, z)$ in the limits $k \downarrow-\frac{1}{6}$ and $|k| \ll 1$ by the methods of [3], obtaining

$$
\begin{aligned}
\lim _{k \downarrow-\frac{1}{6}} \triangle Z(k, z) & =2 \varepsilon \pi(1+b)+O(\varepsilon c)+O\left(\varepsilon^{2}\right), \\
\triangle Z(k, z) & =-\varepsilon \log |k|+6 \varepsilon b+O(-k \log |k|), \text { when }|k| \ll 1, k<0 .
\end{aligned}
$$

By (2.8) the system is obviously cooperating for $b \geq 0$ (and $z$ such that $c z^{2}<1$ if $(2.2)$ or (2.3) are used). By (2.8) and (2.9) we already have a small counteracting region near $\{x=1, y=0\}\left(k=-\frac{1}{6}\right)$ for $b<-1$. As $b$ decreases, from $(2.9-2.10)$, the counteracting region grows until eventually, for $b=O(|\log \varepsilon|)$ and negative, it includes the neighborhood of $W^{\mathrm{u}}(\Gamma)$ and $W^{\mathrm{s}}(\Gamma)\left(\left.\triangle Z(k, z)\right|_{|k| \ll 1}<0\right)$.

The importance of the distinction between $\triangle Z(k, z)>0$ and $\triangle Z(k, z)<0$ becomes clear if one applies the Poincare map $\mathcal{P}$ or $\mathcal{P}^{-1}$ to the curves $P(\Gamma)$ and $P^{-1}(\Gamma)$. The following lemmas proven in [2] describe how $\mathcal{P}$ acts on points at an $O(\varepsilon)$ distance from $P^{-1}(\Gamma)$ (resp. exponentially close to $P^{-1}(\Gamma)$ ), and similarly how $\mathcal{P}^{-1}$ acts on points at an $O(\varepsilon)$ distance from $P(\Gamma)$ (resp. exponentially close to $P(\Gamma)$ ). They will be used frequently throughout this paper, not always with explicit citation.

Lemma 2.1 If $d\left(q_{0}, P^{-1}(\Gamma)\right)=\delta \ll 1$ for a point $q_{0}=(x, 0, z)$ with $k\left(q_{0}\right)<0(+O(\varepsilon))$, then $d\left(\mathcal{P}\left(q_{0}\right), P(\Gamma)\right)=O(\delta)$. Similarly, if $d\left(q_{0}, P(\Gamma)\right)=\delta$ for a point $q_{0}=(x, 0, z)$ with $k\left(q_{0}\right)<0(+O(\varepsilon))$, then $d\left(\mathcal{P}^{-1}\left(q_{0}\right), P^{-1}(\Gamma)\right)=O(\delta)$. 
Here $d(.,$.$) is the standard distance function or, equivalently and in the same order of$ magnitude with respect to $\varepsilon$, the weighted distance expressed in terms of $k$.

In the next lemma $\mathcal{B}$ is a neighborhood of $\Gamma$, independent of $\varepsilon$, in which local (Fenichel) coordinates can be defined.

Lemma 2.2 If a solution of (2.1) enters $\mathcal{B}$ exponentially $\left(O\left(e^{-\kappa / \varepsilon}\right)\right.$ for some constant $\kappa>0)$ close to $W^{\mathrm{s}}(\Gamma)$, the trajectory of this solution remains in $\mathcal{B}$ for an $O\left(\frac{1}{\varepsilon}\right)$ time and leaves $\mathcal{B}$ exponentially close to $W^{\mathrm{u}}(\Gamma)$. Between entering and departing $\mathcal{B}$, a time interval also of length $O\left(\frac{1}{\varepsilon}\right)$ elapses, in which the solution is exponentially close to both $W^{\mathrm{s}}(\Gamma)$ and $W^{\mathrm{u}}(\Gamma)$. During this time interval the $z$ coordinate of the solution changes by an $O(1)$ amount.

Since solutions in the domain of $\mathcal{P}, \mathcal{P}^{-1}$ spend only $O(1)$ time in the fast field (outside $\mathcal{B})$, orbits starting exponentially close to $P^{-1}(\Gamma) \subset W^{\mathrm{s}}(\Gamma)$ enter $\mathcal{B}$ exponentially close to $W^{\mathrm{s}}(\Gamma)$ and orbits leaving $\mathcal{B}$ exponentially close to $W^{\mathrm{u}}(\Gamma)$ pass $P(\Gamma)$ exponentially close. In fact, this observation is a special case of Lemma 2.1. Lemma 2.2 is also a $C^{0}$ version of the $\left(C^{1}\right)$ Exchange Lemma with exponentially small error [11] for this model.

Applying $\mathcal{P}$ and $\mathcal{P}^{-1}$ to points on $P(\Gamma)$ (resp. $P^{-1}(\Gamma)$ ) in the domain of $\mathcal{P}$ (resp. $\mathcal{P}^{-1}$ ) and using these two lemmas, one finds a curve $\mathcal{P}(P(\Gamma))=P^{2}(\Gamma)$ and two distinct curves $\mathcal{P}^{-1}\left(P^{-1}(\Gamma)\right)=P^{-2}(\Gamma)$ as in Figure 1 . This procedure can be repeated by applying $\mathcal{P}$ inside its domain on $P^{2}(\Gamma)$, and $\mathcal{P}^{-1}$ on $P^{-2}(\Gamma)$, etc. The curves $P^{n}(\Gamma) \in W^{\mathrm{u}}(\Gamma)$ are just single tongues, but each $P^{-m}(\Gamma) \in W^{\mathrm{s}}(\Gamma)$ consists of $m$ distinct curves. One of these is a branch "paralleling" $P^{-1}(\Gamma)$, and another is the root tongue of $P^{-m}(\Gamma)$. The others are all parts of haloes surrounding each root tongue $P^{-k}(\Gamma), 1<k<m$. Indeed, for $a>a_{n}^{*}$ and $b>-1$, each tongue $P^{-k}(\Gamma)$ with $k \leq n$ is surrounded by a halo of parallel tongues of $P^{-j}(\Gamma), k+1 \leq j \leq n+1$. See $\S 4$ of [2] and Figure 6 below.

Note that for $b>-1$ the flow in $z$ direction is always upwards for forward time, so the 'tip' of the forward image of a tongue will have greater $z$ coordinate than the tip of the tongue itself. For a detailed description we refer to $§ 4.1$ of [2].

Analyzing the structures of $W^{\mathrm{u}}(\Gamma)$ and $W^{\mathrm{s}}(\Gamma)$ we find more intersections of these manifolds: curves $P^{n}(\Gamma)$ and $P^{-m}(\Gamma)$ intersecting in the neighborhoods of $\gamma^{+}(0)$ and $\gamma^{-}(0)$, see Figure 1. These intersections correspond to homoclinic orbits to $\Gamma$ with multiple loops through the fast field, to be considered further in Section 4. They are created in bifurcations $a_{n}^{*}$, with properties formulated below. The notation $a_{n}^{*}$ is that of [2]. The values $a_{n}^{*}$ depend on $b$, so in fact there is a bifurcation curve $\left\{\left(a_{n}^{*}(b), b\right)\right\}$, which we denote in this paper by

$$
B_{n}(\cdot \underbrace{1}_{2} \underbrace{11 \ldots 1}_{n})
$$

This notation will be explained in $\$ \$ 3.2-4.1 . B_{1}\left(\cdot{ }_{2}^{1}\right)$ is the bifurcation curve on which the stable and unstable manifolds $W^{\mathrm{s}}(\Gamma)$ and $W^{\mathrm{u}}(\Gamma)$ of $\Gamma$ are tangent; $W^{\mathrm{s}}(\Gamma)$ and $W^{\mathrm{u}}(\Gamma)$ intersect in two primary homoclinic orbits for $(a, b)$ to the right of this curve and have no primary intersections for $(a, b)$ to the left of it. Then:

1. For $\left(a^{*}(b), b\right) \in B_{1}\left(\cdot \frac{1}{2}\right)$ there is exactly one homoclinic orbit to $\Gamma$. For $a(b)<a^{*}(b)$, $W^{\mathrm{u}}(\Gamma) \cap W^{\mathrm{s}}(\Gamma)=\emptyset$, and for $a(b)>a^{*}(b)$ there are two single-loop homoclinic orbits. 
2. For each $b$ fixed there exists a sequence of parameter values $a_{n}^{*}$ with $a_{n+1}^{*}>a_{n}^{*}, n>1$, at which two $n$-loop homoclinic orbits are created in a saddle-node bifurcation: for $a<a_{n}^{*} P^{-n}(\Gamma) \cap P(\Gamma)=\emptyset$, while for $a>a_{n}^{*}$ two intersection points $P^{-n}(\Gamma) \cap P(\Gamma)$, corresponding to two $n$-loop homoclinic orbits, exist. In the present notation this ordered sequence of bifurcation curves is called $B_{n}\left(\cdot{ }_{2}^{1} 11 \ldots 1\right)$.

The bifurcations described here occur in both the cooperating and the counteracting cases. We refer to Theorems 4.2, 4.7 and 6.1 of [2].

Remark 2.3 If we replace the equation for $\dot{z}$ by (2.2) the picture changes in that there are now two saddle points $S^{ \pm}$on $\Gamma: S^{+}$with positive and $S^{-}$with negative $z$ coordinate. The point $S^{+}$has a two-dimensional stable manifold $W^{\mathrm{s}}\left(S^{+}\right)$and a one-dimensional strong unstable manifold $W^{\mathrm{u}}\left(S^{+}\right)$, while $S^{-}$has a two-dimensional unstable manifold $W^{\mathrm{u}}\left(S^{-}\right)$ and a one-dimensional strong stable manifold $W^{\mathrm{s}}\left(S^{-}\right)$. Hence the orbit through a point $p \in P^{-1}(\Gamma)$ is homoclinic to $\Gamma$ for $t \rightarrow \infty$ if it tends to $S^{+}$. Orbits through points exponentially close to $p$ will be mapped by $\mathcal{P}$ to a neighborhood of $W^{\mathrm{u}}\left(S^{+}\right) \cap P^{-1}(\Gamma)$ (instead of a point with $z$ coordinate $\gg 1$ as in system (2.1)). Similarly, an orbit homoclinic to $\Gamma$ for $t \rightarrow-\infty$ in system (2.1) corresponds to an orbit originating from $S^{-}$. (See [3], [1] and [6] for a detailed description of the maps $\mathcal{P}$ and $\mathcal{P}^{-1}$ near critical points on $\Gamma$.) The tongues and branches $P^{n}(\Gamma)$ and $P^{-m}(\Gamma)$ are no longer infinitely long as in Figure 1, but are 'pinched' at the intersections of $P^{-1}(\Gamma)$ and $P(\Gamma)$ with the strong stable and unstable manifolds of $S^{ \pm}$. See Figure 4 below and [3].

As $b$ decreases below -1 , near the axis $\{x=1, y=0\}$, the averaged flow in the $z$ direction becomes increasingly negative and tongues $P^{n}(\Gamma)$ and $P^{-m}(\Gamma), m, n>1$ can intersect. First, for $b$ close to -1 , the high order $(n, m \gg 1)$ tongues $P^{n}(\Gamma)$ and $P^{-m}(\Gamma)$ (the latter with its halo) intersect near $\{x=1, y=0\}$, and hence new $(n+m-1)$-loop homoclinic orbits arise. Applying $\mathcal{P}$ and $\mathcal{P}^{-1}$ repeatedly, this implies that $P^{n+m-1}(\Gamma)$ intersects $P^{-1}(\Gamma)$ and $P^{-n-m+1}(\Gamma)$ intersects $P(\Gamma)$. In [2] it was proved that:

1. No intersections of tongues $P^{n}(\Gamma)$ and $P^{-m}(\Gamma)$ can appear before all bifurcations $B_{n}\left(\cdot{ }_{2}^{1} 11 \ldots 1\right)$ have occurred.

2. As $b$ enters the counteracting régime for $a>0$, each tongue $P^{-k}(\Gamma), k \geq 3$, with its halo, bends around $P^{-2}(\Gamma)$ and passes down to $z=-\infty$ before the $P^{-(k-1)}(\Gamma)$ tongue intersects $P(\Gamma)$.

3. The last tongues to pass through $P^{ \pm 1}(\Gamma)$ are $P^{\mp 2}(\Gamma)$.

The orbits created as each tongue and its halo first intersect $P(\Gamma)$ are those described in [2] Section 6, Theorems 6.3-6.9, in which the existence of $4(N-1)-2 N$-loop homoclinic orbits is proved for each $N \geq 2$, with $a>0$ and $b$ sufficiently negative.

Since it is simplest to describe and analyze the flow near $P(\Gamma)$ and $P^{-1}(\Gamma)$, we take $b=O(|\log \varepsilon|)$ and negative. Moreover, in this paper we will show that $\mathcal{P}$ has the structure of a full topological horseshoe only when $b=O(|\log \varepsilon|)$ and sufficiently negative. In this case $P^{\mp 2}(\Gamma)$ and $P(\Gamma)$ intersect as shown in Figure 2 and described in the next result, 

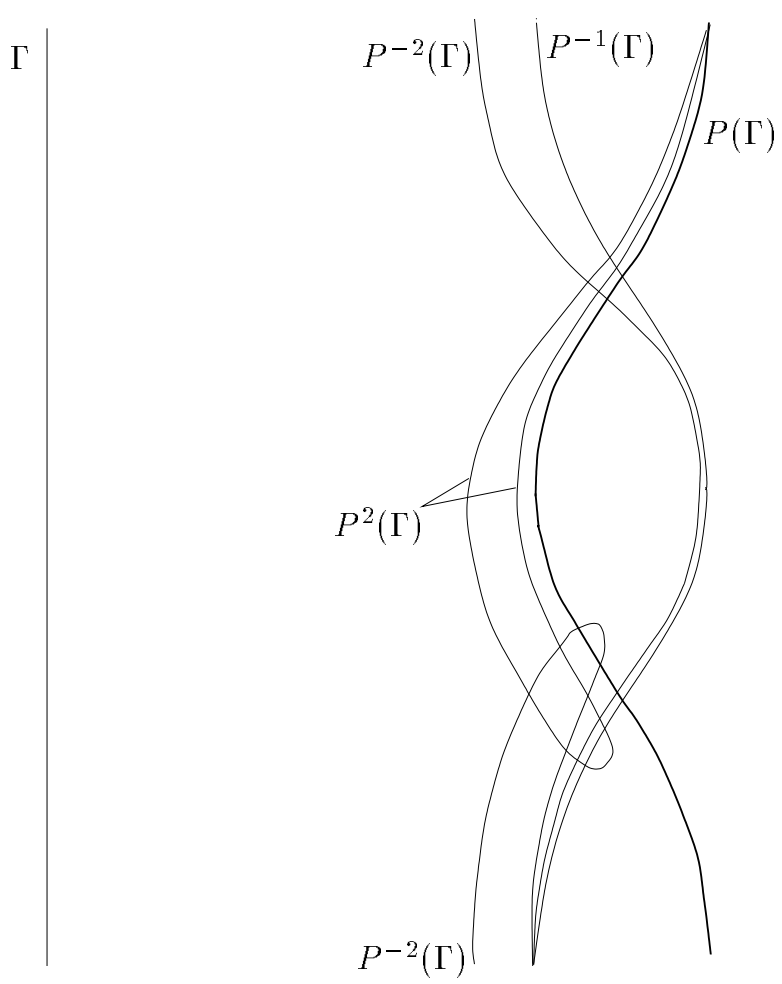

Figure 2: Structure of $W^{\mathrm{u}}(\Gamma)$ and $W^{\mathrm{s}}(\Gamma)$ for $b=O(|\log \varepsilon|)$ sufficiently negative: $P(\Gamma)$ and $P^{-2}(\Gamma)$ (resp. $P^{-1}(\Gamma)$ and $P^{2}(\Gamma)$ ) intersect. Compactified case shown. 


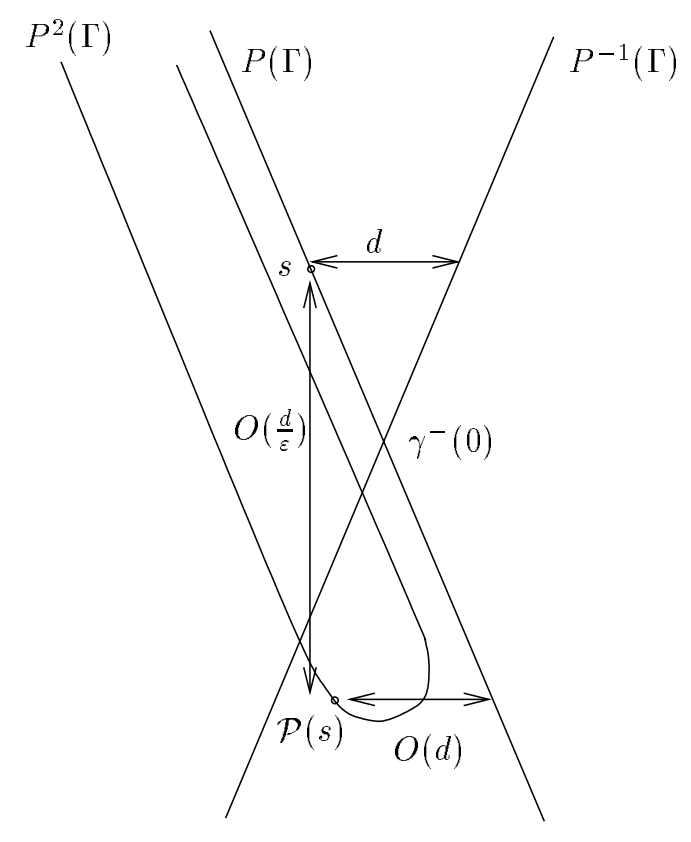

Figure 3: Neighborhood of $\gamma^{-}(0)$ in the plane $\mathcal{V}$, showing conditions on $s \in P(\Gamma)$ to be mapped to the right of $P^{-1}(\Gamma)$ and below $\gamma^{-}(0)$.

which follows from Theorem 6.3 of [2]. Since it is crucial for our subsequent analysis, we give a formulation and proof independent of [2].

Theorem 2.4 For $a=O(1)>0, b=O(|\log \varepsilon|)$, sufficiently negative, the tongues $P^{2}(\Gamma)$ and $P^{-1}(\Gamma)$, and hence $P(\Gamma)$ and $P^{-2}(\Gamma)$, intersect.

Proof: The theorem is proved by showing that there are points in $P(\Gamma)$ that have their images $\mathcal{P}(s) \in P^{2}(\Gamma)$ to the right of $P^{-1}(\Gamma)$ and below $\gamma^{-}(0)$. Without loss of generality we assume $|b| \geq O(1)$ and $b<0$. Take a point $s \in P(\Gamma)$ and define $d=d\left(s, P^{-1}(\Gamma)\right)$ where $d(.,$.$) is the standard distance function (or, equivalently, the distance in k$ ). Assume $d \ll \varepsilon$ is at most algebraically small in $\varepsilon$. Then by Lemma $2.1 d(\mathcal{P}(s), P(\Gamma))=O(d)$. By $\dot{z}=\varepsilon(1+b x)$ (or one of equations (2.2) and (2.3)) and (2.10) the shift in $z$ between $s$ and $\mathcal{P}(s)$ is

$$
\triangle Z(s)=O(\varepsilon b)+O(\varepsilon|\log \varepsilon| ;)
$$

the first term corresponds to the fast flow and is negative if $b$ is negative enough, the second term is the positive slow flow.

If $a=O(1)>0$, the angle between $P(\Gamma)$ and $P^{-1}(\Gamma)$ is of $O(\varepsilon)$. Hence $\mathcal{P}(s)$ lies to the right of $P^{-1}(\Gamma)$ and below $\gamma^{-}(0)$ if $|\triangle Z(s)| \gg \frac{d}{\varepsilon}$ and $\triangle Z(s)<0$; see Figure 3. Combining this with $(2.11)$ we find that $\mathcal{P}(s)$ lies as claimed if

$$
|b| \gg|\log \varepsilon| \text { and } \varepsilon|b| \gg \frac{d}{\varepsilon},
$$

or $|b| \gg \max \left(|\log \varepsilon|, \frac{d}{\varepsilon^{2}}\right)$. Since $d$ is "free" as long as $d \ll \varepsilon$, we can make the special choice $d=O\left(\varepsilon^{2}|\log \varepsilon|\right)$, which ensures that for $|b| \gg|\log \varepsilon|$ and sufficiently negative the 
image of the point $s$ lies to the right of $P^{-1}(\Gamma)$. However, the crucial value of $b$ for which $\triangle Z(s)$ becomes sufficiently negative will be $O(|\log \varepsilon|)$, and we can state the theorem for $|b| \geq|\log \varepsilon|$ sufficiently negative.

\subsection{Existence and local character of periodic orbits}

We consider the compactified vector field, i.e. (2.1) with (2.2). The fixed points of the Poincaré map $\mathcal{P}$ for this vector field are given by

$$
\begin{aligned}
\triangle K(k, z) & =\varepsilon\left(z^{2}-a\right) S_{0}(k)+O\left(\varepsilon^{2}\right)=0 \\
\triangle Z(k, z) & =\varepsilon\left(1-c z^{2}\right) T_{0}(k)+\varepsilon b T_{1}(k)+O\left(\varepsilon^{2}\right)=0,
\end{aligned}
$$

where, for concision, we use the notations for the integrals of $(2.7-2.8)$ introduced in [3]:

$$
\begin{aligned}
& T_{i}(k)=\oint \frac{x^{i}}{\sqrt{2 k+x^{2}-\frac{2}{3} x^{3}}} d x \\
& S_{i}(k)=\oint x^{i} \sqrt{2 k+x^{2}-\frac{2}{3} x^{3}} d x .
\end{aligned}
$$

Since $\triangle K(k, z)=O\left(\varepsilon^{2}\right)$ in the planes $\{z= \pm \sqrt{a}\}, a \geq 0$, and $S_{0}(k) \neq 0$ for $-\frac{1}{6}<k<0$, fixed points can exist only $O(\varepsilon)$ close to these planes. Equating $\triangle Z(k, z)$ to zero in a plane $\left\{z=z_{0}\right\}$ leads to

$$
b=\left(c z_{0}^{2}-1\right) \frac{T_{0}(k)}{T_{1}(k)}+O(\varepsilon)=\frac{c z_{0}^{2}-1}{\mathcal{T}(k)}+O(\varepsilon)
$$

where $\mathcal{T}(k) \stackrel{\text { def }}{=} \frac{T_{1}(k)}{T_{0}(k)}$. If in place of $(2.2)$ we consider $(2.3)$, then $\triangle Z(k, z)=\varepsilon[(1-$ $\left.\left.c z^{2}\right) T_{0}(k)+b T_{1}(k)\right]=0$ for $b=-\frac{1}{\mathcal{T}(k)}$, so we can simply follow the computations for $(2.2)$ with $c=0$.

It was shown in [3] that $\frac{d}{d k} \mathcal{T}(k)<0$ for $-\frac{1}{6}<k<0, \lim _{k \downarrow-1 / 6} \mathcal{T}(k)=1$ and $\lim _{k \uparrow 0} \mathcal{T}(k)=0$. Hence, in the relevant domain $c z_{0}^{2}<1$, for each $b<c z_{0}^{2}-1<0$ there is (to $O(\varepsilon)$ ) exactly one $k(b) \in\left(-\frac{1}{6}, 0\right)$ such that $\triangle Z(k, z)=0$. Thus, for $a>0$ and $b<c a-1<0$ the map $\mathcal{P}$ has two fixed points $\left(k_{0}, \sqrt{a}\right)+$ h.o.t. and $\left(k_{0},-\sqrt{a}\right)+$ h.o.t. which we define $p$ and $q$. These fixed points exist only for $b$ in the counteracting régime; they appear at the axis $\{x=1, y=0\}$ for $b=-1$ and move to the right as $b$ decreases.

To determine the local character of the periodic orbits corresponding to these fixed points, we estimate the linearized map $D \mathcal{P}$. At leading order this yields the matrix:

$$
M=\left.\left(\begin{array}{cc}
1+\frac{\partial}{\partial k} \triangle K & \frac{\partial}{\partial z} \Delta K \\
\frac{\partial}{\partial k} \triangle Z & 1+\frac{\partial}{\partial z} \triangle Z
\end{array}\right)\right|_{\Delta K=\Delta Z=0} \stackrel{\text { def }}{=}\left(\begin{array}{cc}
1+\varepsilon A_{1} & \varepsilon B_{1} \\
\varepsilon C_{1} & 1+\varepsilon D_{1}
\end{array}\right) .
$$

Writing the eigenvalues of $M$ as

$$
\lambda=1+\varepsilon \lambda_{1}+O\left(\varepsilon^{2}\right),
$$


introducing higher order terms $\varepsilon^{2} A_{2}$ end $\varepsilon^{2} D_{2}$ on the diagonal and expanding the characteristic equation we find:

$$
A_{1} D_{1}-B_{1} C_{1}-\left(D_{1}+A_{1}\right) \lambda_{1}+\lambda_{1}^{2}=0
$$

Note that the $O\left(\varepsilon^{2}\right)$ terms $A_{2}$ and $D_{2}$ do not appear at this order. Recall that $\triangle K=0$ only for $z^{2}-a=0$ (to $O(\varepsilon)$ ), so

$$
A_{1}=\left.\frac{1}{\varepsilon} \frac{\partial}{\partial k} \triangle K\right|_{\triangle K=\triangle Z=0}=\left.\left(z^{2}-a\right) \frac{\partial}{\partial k} S_{0}(k)\right|_{\triangle K=\triangle Z=0}=0 .
$$

Using $T_{0}>0, S_{0}>0$ and $z= \pm \sqrt{a}$ for $\triangle K=0$ in the equations

$$
B_{1}=\left.2 z S_{0}(k)\right|_{\triangle Z=0} \text { and } D_{1}=-2 c z T_{0}(k),
$$

we find that

(2.20) $\operatorname{sign} B_{1}=\operatorname{sign} z, \operatorname{sign} D_{1}=-\operatorname{sign} z$ if $c>0$, and $D_{1}=0$ if $c=0$.

Finally, the expressions

$$
\frac{d}{d k} T_{0}=-\frac{6 k T_{0}+T_{1}}{6 k(6 k+1)} \text { and } \frac{d}{d k} T_{1}=\frac{-T_{0}+T_{1}}{6 k+1}
$$

from [3] yield

$$
C_{1}=\left.\left(1-c z^{2}\right)\left(-\frac{6 k T_{0}+T_{1}}{6 k(6 k+1)}\right)\right|_{\triangle K=\triangle Z=0}+\left.b\left(\frac{-T_{0}+T_{1}}{6 k+1}\right)\right|_{\triangle K=\triangle Z=0} .
$$

Since, at leading order, $z^{2}=a$ and $T_{1}=-\frac{1-c a}{b} T_{0}$ for $\triangle Z=0, \triangle K=0$, we can eliminate $T_{1}$ from this expression:

$$
C_{1}=\left.b \frac{Y^{2}-12 k Y-6 k}{6 k(6 k+1)} T_{0}\right|_{\triangle Z=0} \text { with } Y=\frac{1-c a}{b}
$$

The polynomial in $Y$ satisfies $Y^{2}-12 k Y-6 k>0$ for $k \in\left(-\frac{1}{6}, 0\right)$, so

$$
\operatorname{sign} C_{1}=-\operatorname{sign} b .
$$

If $\lambda_{1}^{+}$and $\lambda_{1}^{-}$solve (2.18), then $\lambda_{1}^{+}+\lambda_{1}^{-}=D_{1}$ and $\lambda_{1}^{+} \lambda_{1}^{-}=-B_{1} C_{1}$, since $A_{1}=0$. In the relevant case $b<-1+c a<0$ we can therefore formulate:

Theorem 2.5 For $b<-1+c a<0, c>0,|c| \ll 1$, the periodic orbit corresponding to $q \approx(k(b),-\sqrt{a})$ is unstable $\left(\lambda_{1}^{ \pm}=\phi \pm \omega i\right.$ with $\left.\phi>0\right)$. For $c=0$ it is of center type (at first order). The periodic orbit corresponding to $p \approx(k(b), \sqrt{a})$ is of saddle type for all $b<-1+c a<0, c \geq 0$. 


\section{A topological horseshoe for $b=O(|\log \varepsilon|)$ and negative}

The asymptotic calculations of [2], summarized above in Theorem 2.4, show that for $a=O(1)$ and $b=O(|\log \varepsilon|)$ sufficiently negative, there exists $\varepsilon_{s}(a, b)>0$ such that for all $0<\varepsilon<\varepsilon_{s}(a, b) P(\Gamma)$ and $P^{-2}(\Gamma)$ intersect as shown in Figure 2. Here and in the next section we show that this in turn implies the existence of a topological horseshoe $\Lambda$ whose stable and unstable manifolds intersect the unstable and stable manifolds of $\Gamma$. We use the symbolic dynamics associated with points in $\Lambda$ to construct symbol sequences of arbitrary length that describe homoclinic orbits in $W^{\mathrm{u}}(\Gamma) \cap W^{\mathrm{s}}(\Gamma)$. These sequences precisely characterize orbits created in the subset of homoclinic bifurcations described in [2], and clearly reveal infinitely many additional bifurcations. They also allow us to partially order these bifurcations in terms of their sequences as one moves "monotonically" across the $(a, b)$ parameter plane.

\subsection{Existence of a topological horseshoe and symbolic dynamics}

In the following we sometimes assume that all "primary" intersections of stable and unstable manifolds are transverse and that contraction and expansion estimates for the linearized Poincaré map $D \mathcal{P}$, restricted to certain regions, are sufficient to guarantee hyperbolicity. (We prove this for a model mapping in Section 5.3.) This simplifies our analysis, and implies that the correspondence to shifts on symbol sequences is by homeomorphism. We have been unable to check hyperbolicity by asymptotic calculations of the type done in Section 2, but relaxing this requirement, we still obtain a topological horseshoe and an associated semi-conjugacy to symbol sequences (each sequence has at least one orbit, but possibly more than one, as preimage). Indeed, we have:

Theorem 3.1 For $a(=O(1))>0$ and $b=O(|\log \varepsilon|)$ sufficiently negative, the map $\mathcal{P}$ has an invariant set $\Lambda$ on which $\mathcal{P}$ is semi-conjugate to a full shift on two symbols: $\mathcal{P}$ has a topological horseshoe.

Proof: Theorem 2.4 asserts that the structure of Figure 2 occurs in the parameter range assumed. Using the compactification of Section 2 and "rectifying" this picture, we may construct a region $S$ part of whose image $\mathcal{P}(S) \cap S=V_{1} \cup V_{2}$ lies as shown in Figure 4, such that $\mathcal{P}\left(H_{j}\right)=V_{j}, j=1,2$. Let

$$
\Lambda=\bigcap_{n=-\infty}^{\infty} \mathcal{P}^{n}(S) .
$$

Note that the flow must be compactified to define $\Lambda$, since otherwise the regions $\mathcal{P}^{n}(S)$ might be unbounded in the $z$ direction. The set $\Lambda$ is clearly invariant under $\mathcal{P}$.

As usual in the canonical piecewise linear case ( $\S 5.1$ of [6]) or the more general hyperbolic case ( $\S 3$ of $[15] ; ~ § 5.2$ of [6]), we define a mapping $x \rightarrow \mathbf{a}(x)$ associating each point $x \in \Lambda$ to a bi-infinite sequence $\mathbf{a}(x) \in\{1,2\}^{\mathbf{Z}}$ which describes its itinerary. Specifically, let $\mathbf{a}(x)=\left\{a_{j}(x)\right\}_{j=-\infty}^{\infty}$ with

$$
a_{j}(x)=\left\{\begin{array}{l}
1 \text { if } \mathcal{P}^{j}(x) \in H_{1} \\
2 \text { if } \mathcal{P}^{j}(x) \in H_{2}
\end{array}\right.
$$



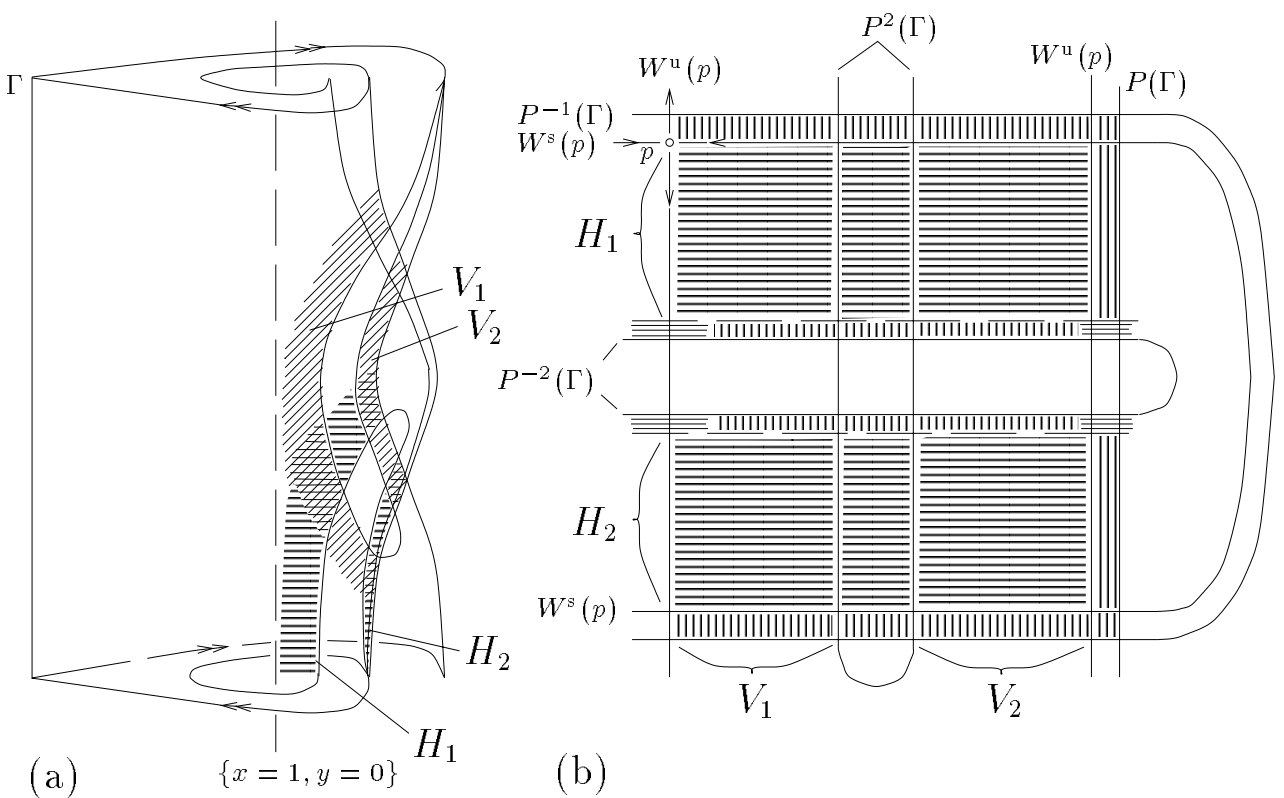

(b)

Figure 4: (a) The compactified flow, showing the regions $H_{j}$ and their images $V_{j}=\mathcal{P}\left(H_{j}\right)$. (b) The topological horseshoe. $S$ is the rectangle bounded by the leftmost and rightmost components of $W_{\mathrm{loc}}^{\mathrm{u}}(p)$, and by the uppermost and lowermost components of $W_{\mathrm{loc}}^{\mathrm{s}}(p) . H_{1}$ and $H_{2}$ are the horizontal strips and $V_{1}$ and $V_{2}$ the vertical strips. $\tilde{H}_{i}$ are the unions of $H_{i}$ and the vertical-dashed regions, $\tilde{V}_{i}$ are constructed likewise.

Clearly each point in $\Lambda$ defines a sequence, for if some iterate $\mathcal{P}^{j}(x) \notin H_{1} \cup H_{2}$, then $x \notin \Lambda$. Moreover, since $V_{j}=\mathcal{P}\left(H_{j}\right)$ is stretched across both $H_{1}$ and $H_{2}$, for every sequence a there exists at least one point $x \in \Lambda$ whose orbit $\mathcal{P}^{j}(x)$ follows a. This gives the desired semiconjugacy to symbol sequences, and thus we have a topological horseshoe.

If $\left.D \mathcal{P}\right|_{\Lambda}$ and $\left.D \mathcal{P}^{-1}\right|_{\Lambda}$ satisfy uniform expansion bounds (cf. $\$ 5.3$ ) the correspondence is unique and $\mathbf{a}$ is a homeomorphism from $\Lambda$ to $\{1,2\}^{\mathbf{Z}}$ equipped with the metric

$$
d(\mathbf{a}, \mathbf{b})=\sum_{j=-\infty}^{\infty} \frac{\left|a_{j}-b_{j}\right|}{2^{|j|}} .
$$

Via the correspondence (3.1), the local component of $W^{\mathrm{s}}(\Lambda)$ containing $x$ is identified by the forward-going part of the sequence $\ldots \cdot a_{0}(x) a_{1}(x) a_{2}(x) \ldots$, while the local component of $W^{\mathrm{u}}(\Lambda)$ is identified by $\ldots a_{-3}(x) a_{-2}(x) a_{-1}(x) \cdot \ldots$. Here we define these local manifolds as $W_{\text {loc }}^{\text {s,u }}(\Lambda)=W^{\text {s,u }}(\Lambda) \cap S$. The symbolic description allows one to enumerate periodic and homo- and heteroclinic points in $\Lambda$, and to prove the existence of a dense orbit [6].

Remark 3.2 The Poincaré map $\mathcal{P}$ is defined "globally" on the cross section $\mathcal{V}=\{y=$ $\left.0,1<x \leq \frac{3}{2}+O(\varepsilon)\right\}$ and the fundamental regions $H_{i}$ and $V_{j}$ are clearly also global in $\mathcal{V}$ : they extend above and below $\gamma^{ \pm}(0)$ (Figure 4(a)). Hence, orbits of (2.1) defined by different sequences $\mathbf{a}(x) \in\{1,2\}^{\mathrm{Z}}$ are not all close in the $(x, y, z)$-phase space (this is clear for the 1-periodic orbits which lie near $z= \pm \sqrt{a}$ ). It is this global aspect that obstructs a 


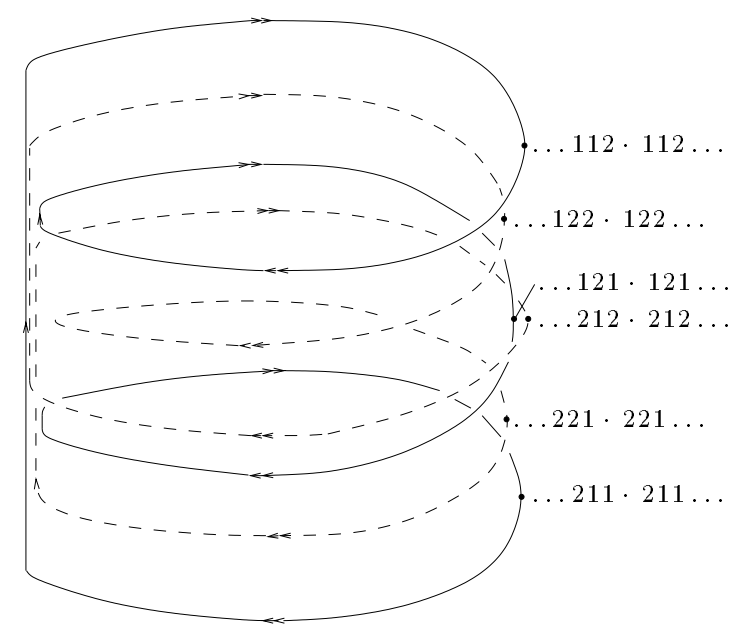

Figure 5: The period three orbits ...112112112 ... and ...122122122 ... in $\Lambda$; the orbits form a nontrivial link.

hyperbolicity proof for $\Lambda$ for the flow itself. To apply Moser's method ([15]; see also $§ 5.3)$, one needs global estimates on the linearizations $D \mathcal{P}$ and $D \mathcal{P}^{-1}$ of the return map. The singular structure of the flow near $W^{\mathrm{u}, \mathrm{s}}(\Gamma)$ is completely different from its regular structure $O(1)$ away from $W^{\mathrm{u}, \mathrm{s}}(\Gamma)$, and it seems difficult to maintain sufficient control on derivatives in matching orbits. (In the formal derivation of a model mapping $F$ of Section 5 , naive matching leads to "unbalanced" orders of $\varepsilon$, cf. §5.1.) Of course this does not mean that $\Lambda$ cannot be hyperbolic, merely that "simple" estimates fail. In the paper [12] by Kopell and Howard a horseshoe was constructed in a three-dimensional system similar to (2.1). However, there - as in most explicit horseshoes constructed in ODEs - the return map is only defined in a small region (of size $O\left(\varepsilon^{2}\right) \times O\left(\varepsilon^{2}\right)$ ). As a consequence, hyperbolicity follows from the linearized flow near a slow manifold. The Šilnikov construction [17], cf. $[6]$ is similarly quasi-local.

Hyperbolic or not, the global nature of $\Lambda$ enables us to find "large" periodic orbits, most of them nontrivially knotted and linked, which encircle the line $\{x=1, y=0\}$ and span the region $z \in[-\sqrt{a},+\sqrt{a}]$. For example, keeping track of twisting as the $H_{i}$ are carried by the flow to $V_{j}$, we can sketch the period three orbits with sequences $\ldots 112112112 \ldots$ and ...122122122 ... as in Figure 5. They are clearly nontrivially linked. (See [5] and references therein for a detailed study of knots and links associated to horseshoes and three-dimensional flows in general.) Such global information on periodic orbits is usually hard to obtain for specific ODEs; here it readily follows from the geometrical construction of $\Lambda$.

Remark 3.3 The existence and stability types of the fixed points $p$ and $q$ proven in $§ 2.3$ are consistent with the horseshoe, since $p(=\ldots 111 \ldots)$ near $z=\sqrt{a}$ is a hyperbolic saddle and $q(=\ldots 222 \ldots)$ near $z=-\sqrt{a}$ is unstable. However, as in the better-known Hénon map [7], one must take parameter ranges such that $q$ has undergone a perioddoubling bifurcation, cf. $\$ 5.1$. In particular, if $\Lambda$ is hyperbolic, the Lambda Lemma (cf. [6], Theorem 5.2.10) implies that the closures of $W^{\mathrm{u}, \mathrm{s}}(p)$ are the stable and unstable manifolds $W^{\mathrm{u}, \mathrm{s}}(\Lambda)$ of $\Lambda$. 


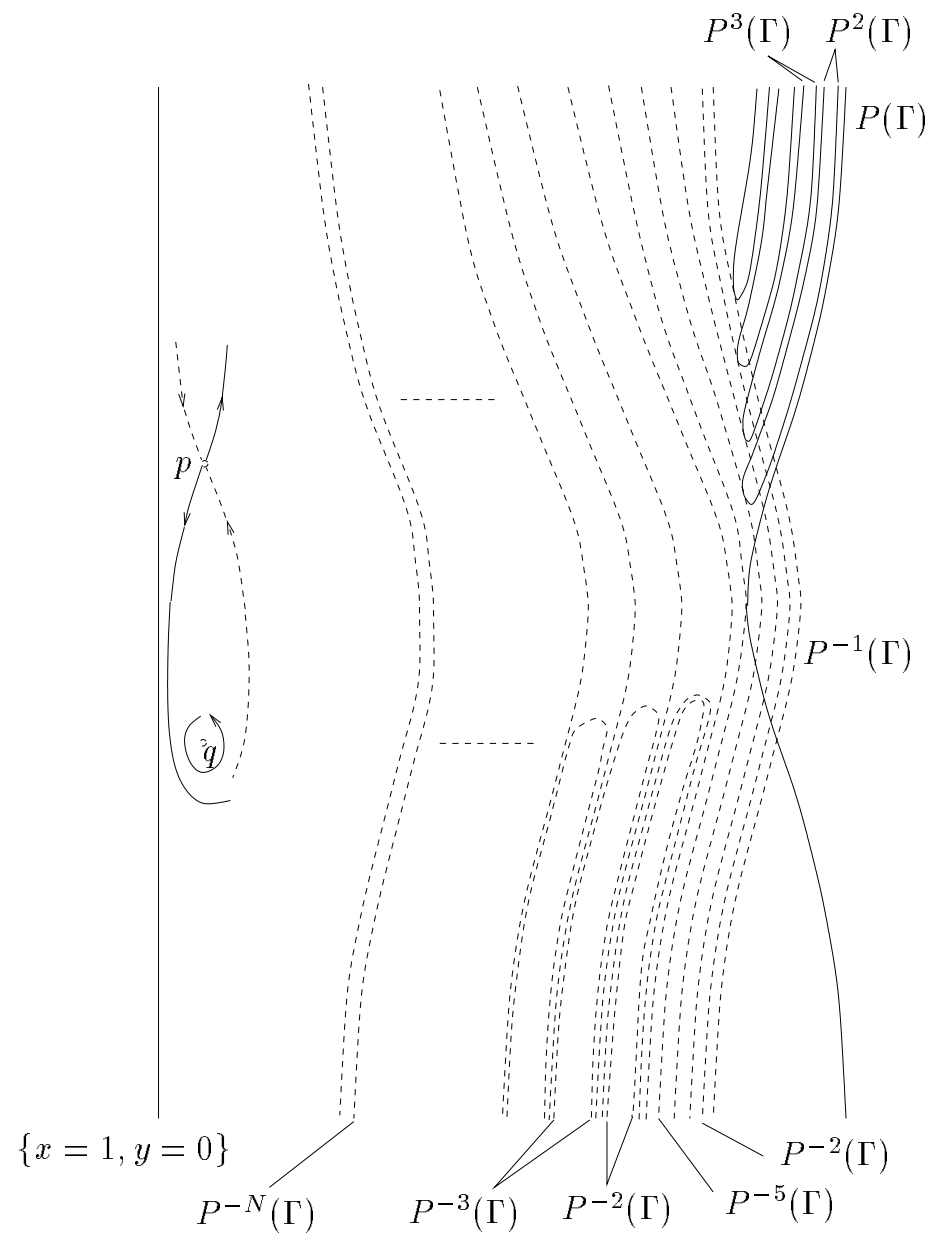

Figure 6: The Poincaré cross section $\{x>1, y=0\}$ showing the fixed points $p$ and $q$, the (local) manifolds $W^{\mathrm{u}, \mathrm{s}}(p)$, and the structure of $W^{\mathrm{u}, \mathrm{s}}(\Gamma)$ at the bifurcation value $a_{5}^{*}=B_{5}\left(\cdot \frac{1}{2} 1111\right)$.

By construction, $p$ lies above all tongues $P^{n}(\Gamma)$ and $W^{\mathbf{u}, \mathrm{s}}(p)$ threads through $W^{\mathbf{u}, \mathrm{s}}(\Gamma)$ (see Figure 4(b)). Carefully studying the structure of this picture and noting that the effect of increasing $b$ is to "draw back" the tongues $P^{n}(\Gamma)$ and $P^{-m}(\Gamma)$, we observe that each tongue $P^{n}(\Gamma) \subset W^{\mathrm{u}}(\Gamma)$ must first intersect $W^{\mathrm{s}}(p)$ before it intersects any tongue $P^{-m}(\Gamma)$ (and $P^{-m}(\Gamma) \subset W^{\mathrm{s}}(\Gamma)$ must similarly intersect $W^{\mathrm{u}}(p)$ before any intersection $P^{n}(\Gamma) \cap P^{-m}(\Gamma)$ occurs). This observation leads to a theorem giving a bound between the cooperating and the counteracting cases. See Figure 6.

Theorem 3.4 In the parameter plane there exists a curve $B_{\infty}\left(\cdot{ }_{2}^{1} 111 \ldots\right)=\left\{\left(a_{\infty}^{*}(b), b\right)\right\}$, such that for every $b$ fixed all bifurcation values $a_{n}^{*}(b)$ satisfy $a_{n}^{*}(b)<a_{\infty}^{*}(b)$ and $W^{\mathrm{s}}(p) \cap$ $W^{\mathrm{u}}(\Gamma)=\emptyset$ for $a<a_{\infty}^{*}(b)$. This curve satisfies

$$
\lim _{b \uparrow-1} a_{\infty}^{*}(b) \begin{cases}=\infty & \text { for } c=0 \\ \leq \frac{1}{c} & \text { for small } c>0\end{cases}
$$




$$
\lim _{b \rightarrow-\infty} a_{\infty}^{*}(b) \ll 1
$$

Corollary 3.5 Since $W^{\mathrm{s}}(p) \cap W^{\mathrm{u}}(\Gamma)=\emptyset$ for $a<a_{\infty}^{*}(b)$, by the above observation there are no intersections between tongues in $W^{\mathrm{u}}(\Gamma)$ and (root) tongues in $W^{\mathrm{s}}(\Gamma)$ for $a<a_{\infty}^{*}(b)$, so the curve $B_{\infty}\left(\cdot{ }_{2}^{1} 111 \ldots\right)$ is a boundary in the parameter plane between the cooperating and the counteracting régimes.

Proof: We consider the cross section $\mathcal{V}=\left\{y=0,1<x<\frac{3}{2}+O(\varepsilon)\right\}$. The fixed point $p$ appears at the axis $\{x=1, y=0\}$ for $b=-1+c a$ (see Section 2.3). At that parameter value the stable and unstable manifolds $W^{\mathrm{u}, \mathrm{s}}(p)$ of $p$ coincide with the invariant line $\{x=1, y=0\}$ and hence $p$ and $W^{\mathrm{u}, \mathrm{s}}(p)$ lie entirely "inside" $W^{\mathrm{u}}(\Gamma)$ and $W^{\mathrm{s}}(\Gamma)$. Since $p$ can never lie on $W^{\mathrm{s}}(\Gamma)$ or $W^{\mathrm{u}}(\Gamma)$, it cannot cross any branch $P^{-n}(\Gamma)$ as $b$ decreases. Hence $p$ remains inside all of them.

When $a<a_{n}^{*}(b)$ for some $b$ fixed, $W^{\mathrm{u}}(\Gamma) \cap P^{-m}(\Gamma)=\emptyset$ for all $m \geq n$ and $W^{\mathrm{u}}(\Gamma)$ lies completely outside $P^{-n}(\Gamma)$. All branches $P^{-m}(\Gamma), m \geq n$ lie between $p$ and $W^{\mathbf{u}}(\Gamma)$. Since $W^{\mathrm{s}}(p)$ cannot intersect $P^{-m}(\Gamma) \subset W^{\mathrm{s}}(\Gamma), W^{\mathrm{s}}(p) \cap W^{\mathrm{u}}(\Gamma)=\emptyset$ for such $a$.

$P(\Gamma)$ must pass through all the branches of $P^{-n}(\Gamma)$ paralleling $P^{-1}(\Gamma)$ before it can intersect any root tongue in $P^{-m}(\Gamma)$ (i.e. all bifurcations $a_{n}^{*}$ or $B_{n}\left(\cdot{ }_{2}^{1} 11 \ldots 11\right.$ ) have occurred before the slow and fast fields become counteracting). Hence there must be an upper bound $a_{\infty}^{*}$ on the bifurcation values $a_{n}^{*}$ for each $\mathrm{b}$ in the counteracting regime. The pairs $\left(a_{\infty}^{*}(b), b\right)$ form a curve $B_{\infty}\left(\cdot{ }_{2}^{1} 111 \ldots\right)$.

As $b \uparrow-1+c a$ the system becomes cooperating, $p$ and $q$ limit on the line $\{x=1, y=0\}$ and the branches $P^{-n}(\Gamma)$ for $n \rightarrow \infty$ remain close to the invariant line $V_{0}=\{x=1, y=0\}$. For $c=0$ this means that $a$ must pass to $\infty$ to make $P(\Gamma)$ intersect all branches $P^{-n}(\Gamma)$; thus $\lim _{b \uparrow-1} a_{\infty}^{*}(b)=\infty$. However, for $c>0$ small, the intersections $P(\Gamma) \cap P^{-1}(\Gamma)$ will cross the strong stable and unstable manifolds of $S^{ \pm}$for $a \approx \frac{1}{c}$. For $a>\frac{1}{c}$ the part of $W^{\mathrm{s}}(\Gamma)$ between the saddles already lies completely outside $W^{\mathrm{u}}(\Gamma)$ (for $a<a^{*} W^{\mathrm{u}}(\Gamma)$ lies outside $W^{\mathrm{s}}(\Gamma)$ !), so all bifurcations $a_{n}^{*}$ or $B_{n}\left(\cdot{ }_{2}^{1} 11 \ldots 11\right)$ must occur before $a$ reaches $\frac{1}{c}$. Thus $\lim _{b \uparrow-1} a_{\infty}^{*}(b) \leq \frac{1}{c}$.

When $b=O(|\log \varepsilon|)<0, P(\Gamma)$ intersects the root tongue of $P^{-m}(\Gamma)$ for every $m$ and all $a=O(1)$. The bifurcations $a_{n}^{*}$ all occur before these tongues first intersect $P(\Gamma)$ and while the $k$ coordinates of $p$ and $q$ are nearly zero (independent of $a$ as long as $a>0$, see (2.16)). Since the tongues $P^{-m}(\Gamma)$ of $W^{\mathrm{s}}(\Gamma)$ and $W^{\mathrm{u}}(\Gamma)$ first intersect for $a \ll 1, W^{\mathrm{s}}(p)$ and $W^{\mathrm{u}}(\Gamma)$ must already intersect for $a \ll 1$. These observations yield $\lim _{b \rightarrow-\infty} a_{\infty}^{*}(b) \ll 1$.

Remark 3.6 One must take care interpreting the limit $a \rightarrow \infty$ : $a$ can be taken large, but it has to remain $O(1)$, since the unperturbed vector field changes dramatically if $a=O\left(\frac{1}{\varepsilon}\right)$, becoming for $\varepsilon=0$ a damped oscillator with no homoclinic manifold. 
Remark 3.7 The relation between bifurcations creating the intersections $W^{\mathrm{u}}(p) \cap W^{\mathrm{s}}(p)$ (and the corresponding standard homoclinic tangle and horseshoe for the map $\mathcal{P}[6]$ ) and the homoclinic bifurcations studied here is quite delicate and interesting. Under appropriate conditions the curve $a=a_{\infty}^{*}(b)$ is also the "first" $W^{\mathrm{u}}(p) \cap W^{\mathrm{s}}(p)$ homoclinic bifurcation curve; this will be the subject of future work.

\subsection{Symbol sequences for orbits in $W^{\mathrm{u}}(\Gamma) \cap W^{\mathrm{s}}(\Gamma)$}

Since $W^{\mathrm{u}}(\Gamma)$ and $W^{\mathrm{s}}(\Gamma)$ thread through $\Lambda$, by construction, an arc of $W^{\mathrm{s}}(\Gamma)$ (resp. $\left.W^{\mathrm{u}}(\Gamma)\right)$ lies between each pair of arcs in $W_{\text {loc }}^{\mathrm{s}}(\Lambda)\left(\operatorname{resp} . W_{\mathrm{loc}}^{\mathrm{u}}(\Lambda)\right)$. We will use this fact to define symbol sequences for points in $W^{\mathbf{u}}(\Gamma) \cap W^{\mathrm{s}}(\Gamma)$ and hence uniquely identify homoclinic orbits to $\Gamma$.

We will need a notion of local manifolds $W_{\text {loc }}^{\text {s,u }}(\Gamma)$, which we define with the help of Figure 4 as $W_{\text {loc }}^{\text {s,u }}(\Gamma)=W^{\text {s,u }}(\Gamma) \cap \tilde{S}$, where $\tilde{S}$ is the rectangle bounded by the leftmost and rightmost components of $W_{\mathrm{loc}}^{\mathrm{u}}(p)$, and by two components of $P^{-1}(\Gamma) ; P_{\mathrm{loc}}^{-k}(\Gamma)$ and $P_{\mathrm{loc}}^{l}(\Gamma)$ are defined analogously. Our construction implies that $P_{\mathrm{loc}}^{-k}(\Gamma)$ consists of $2^{k}$ horizontal arcs and $P_{\mathrm{loc}}^{l}(\Gamma)$ of $2^{l-1}$ vertical arcs. These two sets intersect in exactly $2^{k+l-1}$ points, representing points on $2^{k+l-1}$ distinct $(k+l-1)$-loop homoclinic orbits to $\Gamma$. We will identify these by finite sequences of length $k+l-1$.

We first describe the sequences for the one- and two-loop orbits in $W^{\mathrm{u}}(\Gamma) \cap W^{\mathrm{s}}(\Gamma)$, before proceeding to the general case. We attach the sequences .1 and .2 to the two points $\gamma^{+}(0)$ and $\gamma^{-}(0)$ in $P^{-1}(\Gamma) \cap P(\Gamma)$, identifying the former as lying in the upper component of $P_{\mathrm{loc}}^{-1}(\Gamma)$ and the latter as lying in the lower component of $P_{\mathrm{loc}}^{-1}(\Gamma)$. The four points of $P^{-1}(\Gamma) \cap P^{2}(\Gamma)$ are denoted $1 \cdot 1,2 \cdot 1,1 \cdot 2$ and $2 \cdot 2$, the first pair lying in $P(\Gamma) \cap \tilde{H}_{1}$ and the left (resp. right) component of $P_{\text {loc }}^{2}(\Gamma)$, the second pair lying in $P(\Gamma) \cap \tilde{H}_{2}$ and the left (resp. right) component of $P_{\text {loc }}^{2}(\Gamma)$; their preimages lie in $P^{-2}(\Gamma) \cap P(\Gamma)$ and have sequences $\cdot 11, \cdot 21, \cdot 12$ and $\cdot 22$ respectively. Here $\tilde{H}_{1,2}$ are $H_{1,2}$ extended to the upper (resp. lower) bound $\left(P^{-1}(\Gamma)\right)$ of $\tilde{S}$ and to the lower (resp. upper) part of the root tongue $P^{-2}(\Gamma)$; see Figure 4.

In general, by analogy with (3.1), a point in $P_{\mathrm{loc}}^{-k}(\Gamma) \cap P_{\mathrm{loc}}^{l}(\Gamma)$ is identified by a sequence $b_{-l+1} b_{-l+2} \ldots b_{-1} \cdot b_{0} b_{1} \ldots b_{k-1}$ of length $k+l-1$ in which $b_{-l+1} \ldots b_{-1}$ specifies the component of $P_{\mathrm{loc}}^{l}(\Gamma)$ and $b_{0} \ldots b_{k-1}$ specifies the component of $P_{\mathrm{loc}}^{-k}(\Gamma)$. By construction, shifts of this sequence automatically locate iterates correctly in $P_{\mathrm{loc}}^{-k+j}(\Gamma) \cap P_{\mathrm{loc}}^{l+j}(\Gamma)$. To identify individual orbits, we need only specify one of the $j \in[-l+1, k-1]$ such shifts. To fix notation, we take those points lying in $P_{\mathrm{loc}}^{-(k+l-1)}(\Gamma) \cap P_{\mathrm{loc}}(\Gamma)$, with sequences of the form $\cdot b_{0} b_{1} \ldots b_{k+l-1}$, as representative. The procedure may be summarized as follows. To each point $x \in P_{\mathrm{loc}}^{-(N-1)}(\Gamma) \cap P_{\mathrm{loc}}(\Gamma)$ we assign a sequence $\left\{b_{j}(x)\right\}_{j=0}^{N-2}$ via:

$$
b_{j}(x)=\left\{\begin{array}{l}
1 \text { if } \mathcal{P}^{j}(x) \in W_{\mathrm{loc}}^{\mathrm{u}}(\Gamma) \cap \tilde{H}_{1} \\
2 \text { if } \mathcal{P}^{j}(x) \in W_{\mathrm{loc}}^{\mathrm{u}}(\Gamma) \cap \tilde{H}_{2}
\end{array}\right.
$$

Further examples are given in the next section.

Since arcs of $W^{\mathrm{u}}(\Gamma)$ (resp. $\left.W^{\mathrm{s}}(\Gamma)\right)$ lie between $\operatorname{arcs}$ of $W^{\mathrm{u}}(\Lambda)$ (resp. $W^{\mathrm{s}}(\Lambda)$ ) and $W^{\mathrm{s}}(\Lambda) \cap W^{\mathrm{u}}(\Lambda) \neq \emptyset$, the manifolds $W^{\mathrm{u}}(\Gamma)$ and $W^{\mathrm{s}}(\Lambda)$ (resp. $W^{\mathrm{s}}(\Gamma)$ and $W^{\mathrm{u}}(\Lambda)$ ) must also intersect (in countably many points!). Such intersections correspond to orbits which 


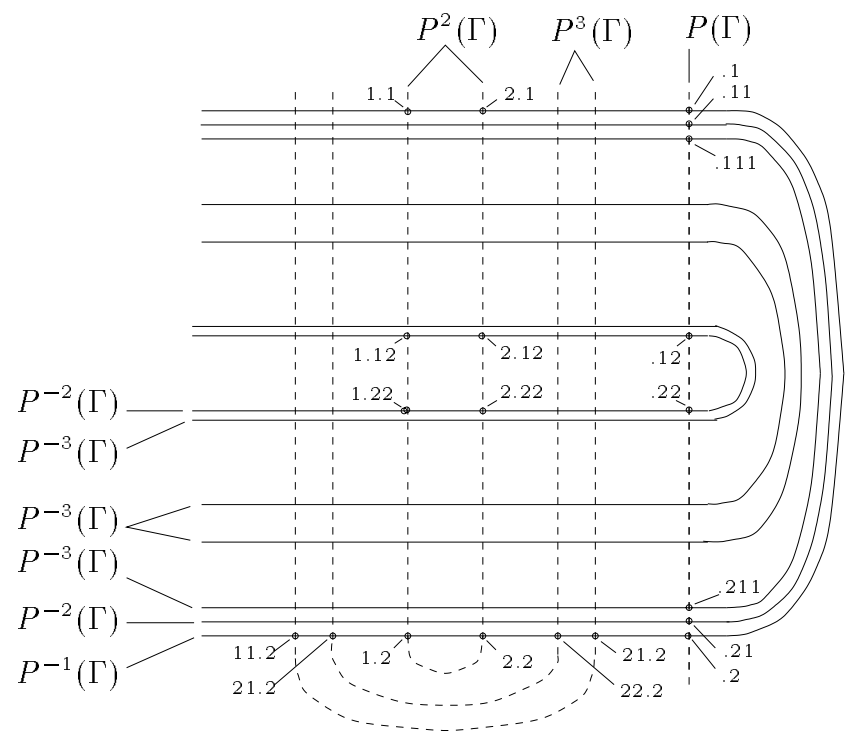

Figure 7: Some sequences for points in $W^{\mathrm{u}}(\Gamma) \cap W^{\mathrm{s}}(\Gamma)$.

originate near $\Gamma$ and first follow $W^{\mathbf{u}}(\Gamma)$, then pass to $\Lambda$ and remain near it for an arbitrarily long 'time', and finally follow $W^{\mathrm{s}}(\Gamma)$ back to $\Gamma$. This provides another interpretation of the codes for homoclinic orbits to $\Gamma$ :

Theorem 3.8 For every finite block $a_{i} \ldots a_{j}$ contained in a sequence $\mathbf{a}(x)$ of a point $x \in \Lambda$ a homoclinic orbit to $\Gamma$ can be constructed. This orbit corresponds to the sequence $\cdot b_{0} \ldots b_{j-i-1}=\cdot a_{i} \ldots a_{j}$ and follows (i.e. remains close to) the orbit of $x$ during a finite time. The beginning and ending points of the sequence represent the intersection points $W^{\mathrm{u}}(\Gamma) \cap W^{\mathrm{s}}(\Lambda)$ and $W^{\mathrm{u}}(\Lambda) \cap W^{\mathrm{s}}(\Gamma)$.

\section{Symbolic dynamics and bifurcation sequences}

In Section 2 certain subsets of homoclinic bifurcations were described. First, for all $b$ (cooperating and counteracting cases) "saddle-node" bifurcations occur, each of which creates a pair of $N$-loop homoclinic orbits having respectively $N$ loops near $z=+\sqrt{a}$, and one loop near $z=-\sqrt{a}$ and $N-1$ near $z=+\sqrt{a}$ ([2], Theorems 4.2 and 6.1 ). For the counteracting $(b<-1)$ case, certain additional homoclinic bifurcations were described ([2], Theorems 6.3-6.9). All these homoclinic orbits belong to $W^{\mathrm{u}}(\Gamma) \cap W^{\mathrm{s}}(\Gamma)$ and may therefore be identified via their symbol sequences. We start by describing the first and simplest, those appearing for all $b$.

\subsection{Identifying homoclinic orbits and bifurcation sequences}

As described in Section 2, the "primary" homoclinic orbits occurring for all $b$ are created when $P(\Gamma)$ first intersects $P^{-N}(\Gamma)$. Since $P(\Gamma)$ penetrates $P^{-1}(\Gamma)$ and the components of $P^{-k}(\Gamma), k \geq 2$, from the right, the homoclinic points necessarily lie in the rightmost 


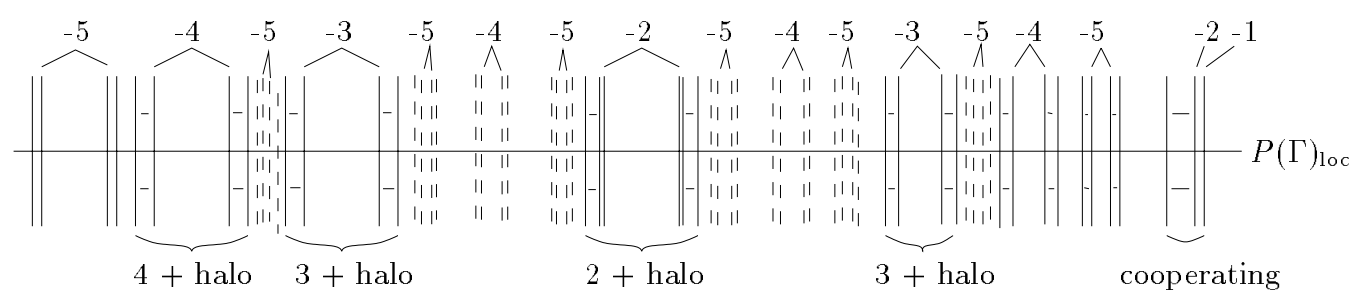

Figure 8: Primary tongues $P_{\mathrm{loc}}^{-k}(\Gamma)$ and their halos intersecting $P_{\mathrm{loc}}(\Gamma), k \leq 5$.

component of $P_{\mathrm{loc}}^{-k}(\Gamma)$ for each $k \geq 2$. The corresponding symbol sequences are therefore

$$
\cdot \underbrace{111 \ldots 11}_{n} \text { and } \cdot 2 \underbrace{11 \ldots 11}_{n-1}
$$

respectively. Sequences of these forms are the only ones to appear in the cooperating case.

To assign further sequences, it is helpful to refer to Figure 8, which shows the tongues $P_{\mathrm{loc}}^{-k}(\Gamma)$ and their halos identified by the integers $k$, with those components relevant to Section 6 of [2] in solid lines and other components dotted. Only tongues with $k \leq 5$ are shown. Using the symbol assignment rule (3.2) of Section 3, we can now write the sequences for $M$-loop orbits created in the "first" intersection of the $N$ "th tongue and its halo with $P(\Gamma)$ (i.e. those described in [2]) as follows:

$$
\begin{aligned}
& N=2 \quad \cdot{ }_{2}^{1} 2 \underbrace{11 \ldots 11}_{M-2},
\end{aligned}
$$

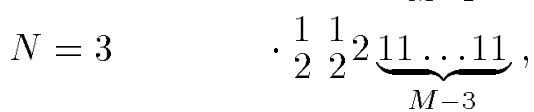

$$
\begin{aligned}
& N=4 \quad \cdot{ }_{2}^{1}{ }_{2}^{1} 2 \underbrace{11 \ldots 11}_{M-4} \text {, } \\
& N=5 \quad \cdot{ }_{2}^{1} 11{ }_{2}^{1} 2 \underbrace{11 \ldots 11}_{M-5} \text {, } \\
& \cdot{ }_{2}^{1} \underbrace{1 \ldots 1}_{N-3} 2_{2}^{1} \underbrace{11 \ldots 11}_{M-N}, \\
& N=M \quad \cdot{ }_{2}^{1} \underbrace{1 \ldots 1}_{M-3} 2_{2}^{1} .
\end{aligned}
$$

Here we adopt the convention of writing $\cdot{ }_{2}^{1} x x \frac{1}{2} x x$ to indicate the quartet of orbits $\cdot 1 x x 1 x x, \cdot 2 x x 1 x x, \cdot 1 x x 2 x x$, and $\cdot 2 x x 2 x x$ associated with a given tongue and its halo. These represent only $4(M-1)$ of the $2^{M}$ possible sequences of length $M$, and hence $M$-loop homoclinic orbits; the remaining ones correspond to the dotted components of $P^{-k}(\Gamma)$ in Figure 8 . We now turn to the question of how and in what order(s) these orbits appear as the parameters $a, b$ vary. 


$$
\begin{aligned}
& B_{1}\left(\cdot \frac{1}{2}\right) \sim a^{*}, \quad B_{1}(\cdot \cdot_{2}^{1} \underbrace{11 \ldots 1}_{N-1}) \sim a_{N}^{*}, \quad B_{\infty}\left(\cdot \cdot_{2}^{1} 111 \ldots\right) \sim a_{\infty}^{*}, \\
& B_{2}\left(\cdot{ }_{2}^{1} 2\right) \sim b_{1,2}^{i}, \quad B_{3}\left(\cdot \cdot_{2}^{1} 2_{2}^{1} 2\right) \sim b_{2,2}^{i, i i}=b_{2}^{i, i i}, \quad B_{4}\left(\cdot \cdot_{2}^{1} 1_{2}^{1} 2\right) \sim b_{2,3}^{i, i i}, \\
& B_{N}(\cdot{ }_{2}^{1} \underbrace{11 \ldots 1}_{N-3} 1_{2}^{1} 2) \sim b_{\frac{N+1}{2}}^{i, i i}(N \text { odd }), \sim b_{\frac{N}{2}, \frac{N+1}{2}}^{i, i i}(N \text { even }) .
\end{aligned}
$$

Table 1: Equivalences of bifurcation notations (see text).

In [2] and Section 2.3 above we described how the $N$-loop homoclinic orbits with sequences $\cdot{ }_{2}^{1} 11 \ldots 11$ appear as $a$ increases for fixed $b$. These bifurcations, in which $P(\Gamma)$ passes through the rightmost components of $P^{-N}(\Gamma), N \geq 2$, must take place before further homoclinic orbits to $\Gamma$ can appear. Then with $a(=O(1))>0$ fixed and decreasing $b$ and for each $N \geq 2$, additional bifurcations occur in which the other $4(N-1)-2 N$-loop orbits identified in (4.1) are created.

The key to understanding the bifurcation set in which these and the balance of the $2^{N} N$-loop orbits appear, is to use the Cantor set-like structure of the countable set $\bigcup_{k=1}^{\infty} P_{\mathrm{loc}}^{-k}(\Gamma)$ (see Figure 8) to define bifurcation curves $B_{N}\left(\cdot b_{0} \ldots b_{N-1}\right)$ in $(a, b)$ space on which $N$-loop orbits with sequences $\cdot b_{0} \ldots b_{N-1}$ appear. For the reader's convenience, we provide in Table 1 a translation from the present notation to the $a_{n}^{*}, b_{n, n+1}^{i, i i}$ and $b_{n}^{i, i i}$ notation used in [2]. The main result of [2] concerning bifurcation sequences is Theorem 6.6 , which establishes the order $b_{n-1, n}^{i}<b_{n}^{i i}<b_{n}^{i}<b_{n, n+1}^{i i}<b_{n, n+1}^{i}, \forall n>1$. In the present terms this is:

$$
\begin{aligned}
B_{2}\left(\cdot{ }_{2}^{1} 2\right)<B_{3}\left(\cdot \cdot_{2}^{1}{ }_{2}\right. & 2)<\ldots<B_{N}(\cdot \cdot_{2}^{1} \underbrace{1 \ldots 1}_{N-3}{ }_{2}^{1} 2)< \\
& <B_{N+1}(\cdot{ }_{2}^{1} \underbrace{1 \ldots 1}_{N-2}{ }_{2}^{1} 2)<\ldots<B_{\infty} .
\end{aligned}
$$

Here the notation $<$ means that the curve $B_{N+1}\left(\cdot{ }_{2}^{1} \ldots 1_{2}^{1} 2\right)$ lies above $B_{N}\left(\cdot{ }_{2}^{1} \ldots{ }_{2}^{1} 2\right)$ in the $(a, b)$ plane. Here, as above, $\cdot{ }_{2}^{1} x x$ (respectively $\cdot{ }_{2}^{1} x x{ }_{2}^{1} x x$ ) denotes a pair (respectively quartet) of orbits and in writing $B_{N}\left(\cdot \frac{1}{2} x x \frac{1}{2} x x\right)$ we do not distinguish between the first and second bifurcations $\left(b_{n}^{i}, b_{n}^{i i}\right)$ in which such a quartet is born. Indeed, as shown in $[9,8]$, the genealogies may change in a two-parameter problem such as the present one. This exactly corresponds to what is sketched in $\S 6.4$ of [2]: $\cdot{ }_{2}^{1} x x{ }_{2}^{1} x x$ are generically created pairwise as either $\cdot 1 x x{ }_{2}^{1} x x$ followed by $\cdot 2 x x{ }_{2}^{1} x x$ or $\cdot{ }_{2}^{1} x x 1 x x$ followed by $\cdot{ }_{2}^{1} x x 2 x x$. Thus, $B_{N}\left(\cdot{ }_{2}^{1} x x{ }_{2}^{1} x x\right)$ denotes a pair of saddle-node bifurcations. 
As noted in Theorem 6.8 of [2], four additional $M$-loop orbits are created for each $M>N$ as the root tongue $P^{-N}(\Gamma)$ passes through $P(\Gamma)$. These form a halo around each of the leftmost and rightmost pairs of components of $P_{\mathrm{loc}}^{-N}(\Gamma)$ (cf. Figure 8), and referring to (4.1) we may identify their bifurcation sets as:

$$
B_{M}(\cdot{ }_{2}^{1} \underbrace{1 \ldots 1}_{N-3}{ }_{2}^{1} 2 \underbrace{11 \ldots 11}_{M-N}) .
$$

In addition to (4.2) we can write

$$
\begin{gathered}
B_{\infty}\left(\cdot{ }_{2}^{1} 111 \ldots\right)<\ldots<B_{N+1}(\cdot{ }_{2}^{1} \underbrace{11 \ldots 1}_{N})<B_{N}(\cdot{ }_{2}^{1} \underbrace{11 \ldots 1}_{N-1})<\ldots \\
<B_{2}\left(\cdot \cdot_{2}^{1} 1\right)<B_{1}\left(\cdot \cdot_{2}^{1}\right), \\
B_{2}\left(\cdot{ }_{2}^{1} 2\right)<B_{3}\left(\cdot \cdot_{2}^{1} 21\right)<\ldots<B_{N}(\cdot{ }_{2}^{1} \underbrace{11 \ldots 1}_{N-2})< \\
<B_{N+1}(\cdot \cdot_{2}^{1} \underbrace{11 \ldots 1}_{N-1})<\ldots<B_{\infty}\left(\cdot \cdot_{2}^{1} 2111 \ldots\right),
\end{gathered}
$$

to specify the orders in which pairs of orbits are created in the first (cooperating) and final bifurcation sequences up to the bifurcation $B_{2}\left(\cdot{ }_{2}^{1} 2\right)$ in which the (topological) horseshoe is completed. This final bifurcation sequence involves the tongue $P^{-2}(\Gamma)$ and its entire halo. Note that (4.3) corresponds to the ordering $a^{*}<a_{2}^{*}<a_{3}^{*}<\ldots<a_{\infty}^{*}$ of [2] (the curve $a_{N-1}^{*}$ lies to the left of $a_{N}^{*}$ in the $(a, b)$ plane, or in terms of $B_{N}(\ldots)$ the curve $B_{N-1}\left(\cdot{ }_{2}^{1} 11 \ldots 1\right)$ lies above $\left.B_{N}\left(\cdot{ }_{2}^{1} 11 \ldots 1\right)\right)$. For $\varepsilon$ small enough (certainly for $\varepsilon<\varepsilon_{s}(a, b)$, cf. Section 3) the orderings (4.2), (4.3) and (4.4) combined with Theorem 3.4 imply the bifurcation diagram sketched in Figure 9.

This picture is primarily qualitative, but some quantitative results may also be noted. Theorem 5.1 of [2] states that $a_{n+1}^{*}-a_{n}^{*}=O\left(\varepsilon^{2}(\log \varepsilon)^{2}\right)$ for $n=O(1)$ and $b>-1$. Then by Theorem 5.3 of [2] $a_{n+1}^{*}=a_{n}^{*}+O\left(a_{n}^{*}\right)$ for $n>O(1)$. Moreover, since the complete topological horseshoe $\Lambda$ exists for all $b=O(|\log \varepsilon|)$ sufficiently negative, $a=O(1)$, the final bifurcation curve $B_{2}\left(\cdot{ }_{2}^{1} 2\right)$ satisfies

$$
\lim _{b \downarrow-\infty} a(b)=o(1) \text { for } a(b) \in B_{2}\left(\cdot{ }_{2}^{1} 2\right),
$$

and hence all other curves $B_{N}(\ldots)$ must approach a similar limit. On the other hand, by Theorem 3.4 the curve $B_{\infty}\left(\cdot{ }_{2}^{1} 111 \ldots\right) \sim a_{\infty}^{*}$ is an upper bound for all counteracting bifurcation curves, since no root tongue $P^{-N}(\Gamma)$ can intersect $P(\Gamma)$ before $P(\Gamma)$ has passed all branches $P^{-M}(\Gamma), M \geq 1$, and hence the bifurcation $B_{\infty}\left(\cdot{ }_{2}^{1} 11 \ldots\right)$ has occurred. 


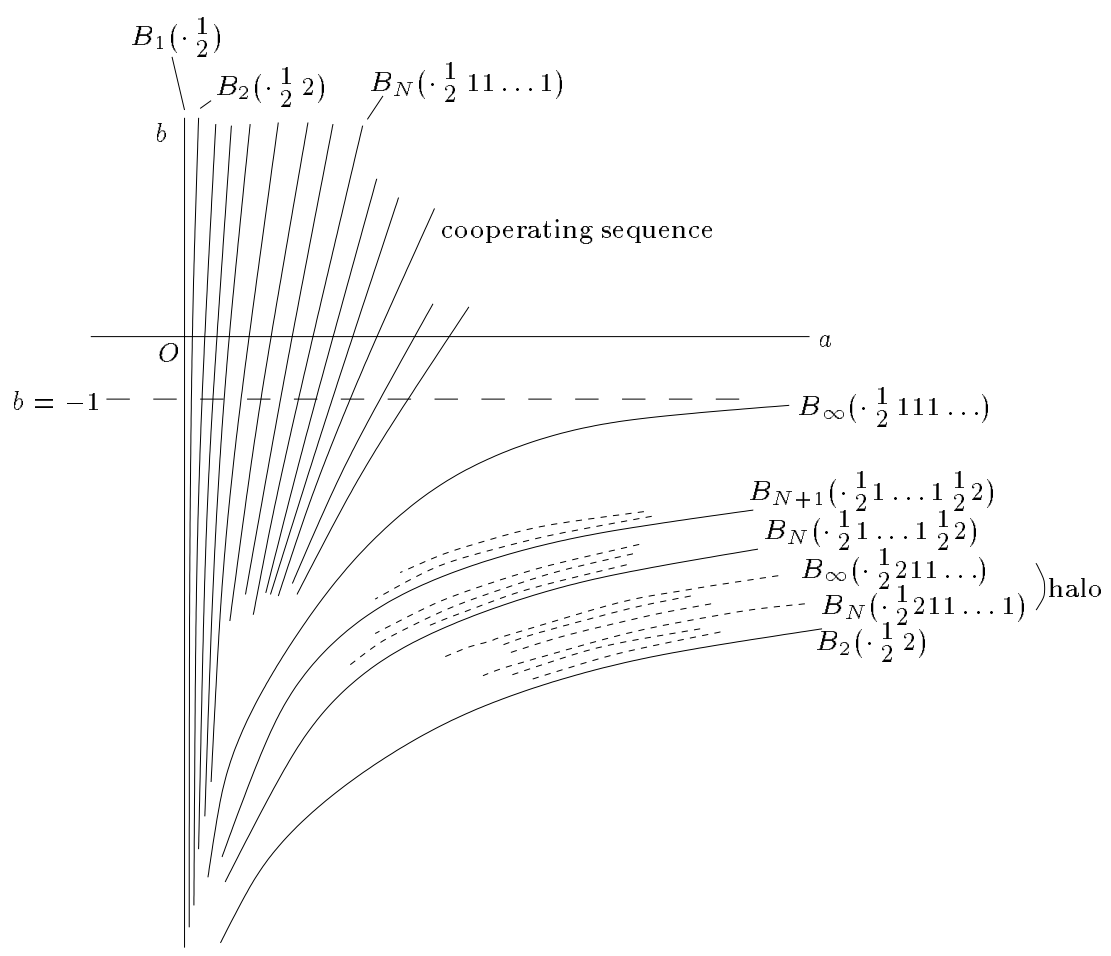

Figure 9: Partial bifurcation diagram in $(a, b)$-plane. The halo bifurcations are indicated by the dotted lines.

One can give some additional partial orderings by noting that all members of the halo of any root tongue of $P^{-N}(\Gamma)$ must first intersect $P(\Gamma)$ before the root tongue itself: specifically, if two branches in the halo belong to $P^{-k}(\Gamma)$ and $P^{-l}(\Gamma), k>l$, then $P^{-k}(\Gamma)$ already intersects $P(\Gamma)$ twice before intersections $P^{-l}(\Gamma) \cap P(\Gamma)$ appear. However, the halo bifurcations may be "completed" after the second root tongue bifurcation or between the first and second root tongue bifurcations. See the examples for $P^{-3}(\Gamma)$ in Figures 10(a), where

$$
B_{5}\left(\cdot{ }_{2}^{1} 211\right)>B_{4}\left(\cdot 1{ }_{2}^{1} 21\right)>B_{3}\left(\cdot 1 \frac{1}{2} 2\right)>B_{5}\left(\cdot 22_{2}^{1} 211\right)>B_{4}\left(\cdot 2_{2}^{1} 21\right)>B_{3}\left(\cdot 22_{2}^{1}\right)
$$

and (b), where

$$
B_{5}\left(\cdot{ }_{2}^{1} 1211\right)>B_{4}\left(\cdot{ }_{2}^{1} 121\right)>B_{3}\left(\cdot{ }_{2}^{1} 12\right)>B_{3}\left(\cdot{ }_{2}^{1} 22\right)>B_{4}\left(\cdot{ }_{2}^{1} 221\right)>B_{5}\left(\cdot{ }_{2}^{1} 2211\right) .
$$

A mix between both sequences may also occur, for instance

$$
B_{5}\left(\cdot{ }_{2}^{1} 1211\right)>B_{4}(\text { first })>B_{3}\left(\cdot 1_{2}^{1} 2\right)>B_{4}(\text { second })>B_{5}\left(\cdot{ }_{2}^{1} 2211\right)>B_{3}\left(\cdot 2_{2}^{1} 2\right) .
$$

Here in $B_{4}$ (first) the orbit $\cdot 1121$ and a preliminary state of the other three orbits are created, and in $B_{4}$ (second) the preliminary state bifurcates to the orbits $\cdot 1221, \cdot 2121$ and . 2221 by a third order contact, see Figure 10(c).

Generically, there are two other possible bifurcation sequences (or a mix of them), but exponential closeness of the haloes evidently precludes their appearance here. In $§ 5.2$ we 
(a)
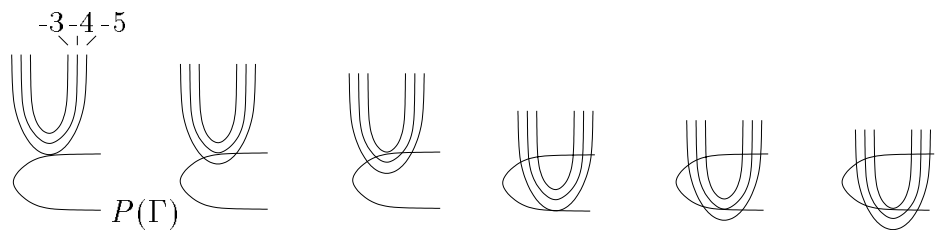

(b)
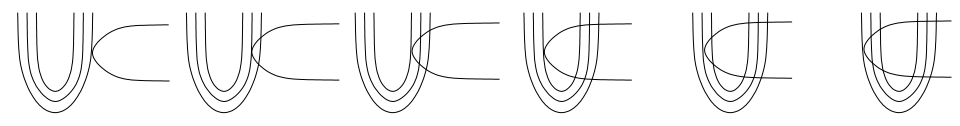

(c)
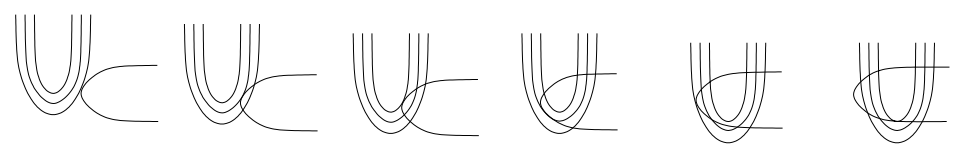

Figure 10: Examples of different admissible sequences.

will find that a 'mixed scenario' with cubic tangencies, as shown in in Figure 10(c), occurs at leading order for the bifurcations $B_{3}\left(\cdot \begin{array}{ll}1 & 1 \\ 2 & 2\end{array} 2\right)$ of a model mapping. There the higher order corrections restore the generic scenarios of Figures 10(a) and (b).

\subsection{Horseshoes for $\mathcal{P}^{N}$ with $N>1$}

In this subsection we construct a horseshoe $\Lambda^{N}$ for the $N$ th iterate $\mathcal{P}^{N}$ of $\mathcal{P}$. The existence of $\Lambda^{N}$ for large $N$ ensures horseshoe behavior throughout the counteracting régime, including the physically relevant case $a, b=O(1)$ (and $a>0, b<-1$ ).

After passing either the bifurcation $B_{N+1}\left(\cdot{ }_{2}^{1} 11 \ldots 112\right)$ or $B_{N+1}\left(\cdot 11 \ldots 11 \frac{1}{2} 2\right)$, depending on the ordering, the root tongue $P^{-N-1}(\Gamma)$ and its entire halo intersect $P(\Gamma)$. In a manner analogous to Figure 4 we can define strips $H_{j}^{N}$ and their images $V_{j}^{N}=\mathcal{P}^{N}\left(H_{j}^{N}\right)$ under $\mathcal{P}^{N}$ such that $\Lambda^{N}=\bigcup_{j=-\infty}^{\infty} \mathcal{P}^{j N}\left(H_{1}^{N} \cup H_{2}^{N}\right)$ is a topological horseshoe for the $N$ th iterate $\mathcal{P}^{N}$ of $\mathcal{P}$ :

Theorem 4.1 As soon as two $(N+1)$-pulse homoclinic orbits are created in one of the bifurcations $B_{N+1}\left(\cdot{ }_{2}^{1} 11 \ldots 112\right)$ or $B_{N+1}\left(\cdot 11 \ldots 11{ }_{2}^{1} 2\right)$ the map $\mathcal{P}^{N}$ has an invariant set $\Lambda^{N}$ on which $\mathcal{P}^{N}$ is semi-conjugate to a full shift on two symbols: $\mathcal{P}^{N}$ has a topological horseshoe.

Sketch of Proof: Figure 11 illustrates the construction of $\Lambda^{N}$. The structure of these horseshoes is not as clean as that of $\Lambda$ for $\mathcal{P}$, although the proof proceeds analogously. The "strips" $H_{2}^{N}$ and $V_{2}^{N}$ are invaded by tongues $P^{-k}(\Gamma)$ (resp. $P^{k}(\Gamma)$ ), $1<k \leq N$ that are cut out by iterates $\mathcal{P}^{ \pm k}, 1 \leq k<N$. Moreover, if we define symbol sequences similar to $(3.1)$ :

$$
a_{j}^{N}(x)=\left\{\begin{array}{l}
1^{N} \text { if } \mathcal{P}^{j N}(x) \in H_{1}^{N} \\
2^{N} \text { if } \mathcal{P}^{j N}(x) \in H_{2}^{N}
\end{array}\right.
$$




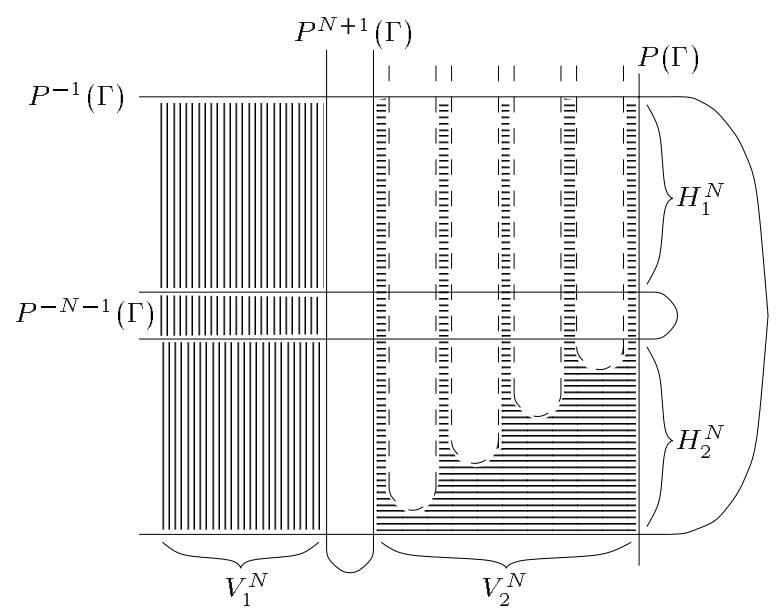

Figure 11: Regions $H_{j}^{N}$ and their images $V_{j}^{N}=\mathcal{P}^{N}\left(H_{j}^{N}\right)$ when the tongue $P^{-N-1}(\Gamma)$ just intersects $P(\Gamma)$.

the correspondence between points $x \in \Lambda^{N}$ and sequences $\mathbf{a}^{N}(x)$ is certainly non-unique: there are sequences with more than one preimage, and thus the horseshoe cannot be hyperbolic.

However, we can determine some specific symbol sequences in $\Lambda^{N}$ as a subset of those in $\Lambda^{1}=\Lambda$. The correspondence (4.5) is not convenient for this, and we shall label points in $\Lambda^{N}$, like points in $\Lambda$, by sequences $\mathbf{a}(x)$ defined as in (3.1). Now these sequences cannot be defined in general when the tongue $P^{-N-1}(\Gamma)$ intersects $P(\Gamma)$ but $P^{-N}(\Gamma)$ does not, for the strips $H_{k}$ and $V_{l}, k, l=1,2$ cannot yet be defined, cf. [9]. Nonetheless, if we apply $\mathcal{P}^{N+1}$ to the largest possible horizontal strip, $H_{1}^{N}=S^{N}$, we easily find that $\mathcal{P}^{N+1}\left(S^{N}\right)$ intersects $S^{N}$ in two disjoint strips, showing that $\mathcal{P}^{N+1}$ has a 2-shift.

Indeed, following the proof of the Smale-Birkhoff Homoclinic Theorem and defining neighborhoods $U$ and $V$ as in [6] (Theorem 5.3.5), we obtain a transition matrix $A$ which enables us to symbolically describe points in $\bigcap_{j=-\infty}^{\infty} \mathcal{P}^{(N+1) j}\left(S^{N}\right) \subset \Lambda^{N+1}$, especially the periodic points. For $N$ odd we define two squares $U$ and $V \subset\left(\mathcal{P}^{-\left(\frac{N-1}{2}\right)}(U) \cap \mathcal{P}^{\frac{N+3}{2}}(U)\right)$ such that $U$ contains all points $x$ that have sequences $\mathbf{a}(x)$ with a central block

$$
\underbrace{11 \ldots 1}_{\frac{N-1}{2}} \cdot \underbrace{1 \ldots 11}_{\frac{N+1}{2}}
$$

and likewise $V$ contains points with sequences with central block

$$
\underbrace{11 \ldots 1}_{N} 2 \cdot \underbrace{1 \ldots 11}_{N}
$$

Since $V$ is a subset of $\mathcal{P}^{-\left(\frac{N-1}{2}\right)}(U) \cap \mathcal{P}^{\frac{N+3}{2}}(U)$ that does not intersect $U$, this gives a collection $\left\{U, \mathcal{P}^{-\left(\frac{N+1}{2}\right)}(V), \ldots, \mathcal{P}^{\frac{N-3}{2}}(V)\right\}$ of disjoint sets which forms a Markov partition 
$\mathcal{R}$ for a zero-dimensional invariant set. In the same way we define for $N$ even a set $U$ containing all points with a central block

$$
\underbrace{11 \ldots 1}_{\frac{N}{2}} \underbrace{1 \ldots 11}_{\frac{N}{2}}
$$

in their sequences, $V \subset\left(\mathcal{P}^{-\left(\frac{N}{2}\right)}(U) \cap \mathcal{P}^{\frac{N}{2}+1}(U)\right)$ containing the points with a middle block

$$
\underbrace{11 \ldots 1}_{N} 2 \cdot \underbrace{1 \ldots 11}_{N}
$$

and a collection $\mathcal{R}=\left\{U, \mathcal{P}^{-\left(\frac{N}{2}\right)}(V), \ldots, \mathcal{P}^{\frac{N}{2}-1}(V)\right\}$

In both cases the $(N+1) \times(N+1)$ transition matrix $A$ is

$$
\left(\begin{array}{ccccc}
1 & 1 & 0 & \ldots & 0 \\
0 & 0 & 1 & \ldots & 0 \\
\vdots & \vdots & \vdots & & \vdots \\
0 & 0 & 0 & \ldots & 1 \\
1 & 0 & 0 & \ldots & 0
\end{array}\right)
$$

Now trace $\left(A^{k}\right)$ is equal to the number of fixed points for $\mathcal{P}^{k}$. For $0<k<N+1$ $\operatorname{trace}\left(A^{k}\right)=1$, so the only fixed point is

$$
\underbrace{11 \ldots 1}_{k} \text {. }
$$

As expected $\operatorname{trace}\left(A^{N+1}\right)=N+2$; there are $N+2 \operatorname{period}-(N+1)$ points: the fixed point $11 \ldots 1$ and the points in the period- $(N+1)$ orbit $11 \ldots 12 \mapsto 11 \ldots 121 \mapsto \ldots \mapsto 211 \ldots 1$. Thus we find not only period- $j(N+1)$ points $(j \geq 1)$, but also a period- $k$ orbit for all $k>N+1$. Such a (nontrivial) orbit is for instance found by "marking time" at $U$ for $k-(N+1)$ steps, then successively following the iterates $\mathcal{P}^{n}(V) \in \mathcal{R}$ and returning to $U$. Its sequence is

$$
\underbrace{11 \ldots 1}_{k-1} 2 \text { periodically repeated. }
$$

Finally, we see that every sequence which has at least $N$ 1's between each pair of 2's, occurs in $\Lambda^{N+1}$.

\section{A model for the Poincaré map $\mathcal{P}$}

In this section we formally derive and analyze an explicit model $F$ for the Poincaré return map $\mathcal{P}$. We start with the derivation in $\$ 5.1$, then construction of a topological horseshoe and explicit bifurcation calculations for small $\varepsilon$ follow in $§ 5.2$, and finally we give a hyperbolicity proof for large $\varepsilon$ in $§ 5.3$. 


\subsection{Derivation of the model mapping}

As in Šilnikov [17], c.f. [6], we write $F=S_{10} \circ T_{01}$ as the composition of a local map $T_{01}$ obtained by integrating the flow near $\Gamma$ in Fenichel coordinates, and a global map $S_{01}$ chosen as a simple polynomial to capture the leading order behavior near the quadratic tangency of $W^{\mathrm{u}}(\Gamma)$ and $W^{\mathrm{s}}(\Gamma)$. Specifically, $T_{01}$ is approximated by the linearized system

$$
\left\{\begin{array}{l}
\dot{p}=p \\
\dot{q}=-q \\
\dot{z}=\varepsilon,
\end{array}\right.
$$

integrated from the local cross section $\Sigma_{0}=\{(p, q, z) \mid q=\Delta\}$ to $\Sigma_{1}=\{(p, q, z) \mid p=\Delta\}$. Letting $\left(k_{0}, z_{0}\right) \in \Sigma_{0}$ and $\left(k_{1}, z_{1}\right) \in \Sigma_{1}$ denote initial and "final" conditions, this yields

$$
T_{01}\left(k_{0}, z_{0}\right)=\left(k_{1}, z_{1}\right)=\left(k_{0}, z_{0}+\varepsilon \log \left(\frac{\Delta}{k_{0}}\right)\right) .
$$

The global map $S_{10}: \Sigma_{1} \rightarrow \Sigma_{0}$ is derived by assuming that vertical components are carried down by an amount $\varepsilon B$, mimicking the counteracting effect of the flow for $x>-\frac{1}{b}$, and that vertical line segments $k_{1}=c \geq 0$ are carried into parabolas $k=\varepsilon\left(A-z^{2}\right)+c$. This leads to

$$
S_{10}\left(k_{1}, z_{1}\right)=\left(k_{2}, z_{2}\right)=\left(k_{1}+\varepsilon\left[A-\left(z_{1}-\varepsilon B\right)^{2}\right], z_{1}-\varepsilon B\right),
$$

and so the composed "logarithmic-quadratic" map may be written

$$
F\left(k_{0}, z_{0}\right)=\left(k_{0}+\varepsilon\left[A-\left(z_{0}+\varepsilon f\left(k_{0}\right)\right)^{2}\right], z_{0}+\varepsilon f\left(k_{0}\right)\right) ; f\left(k_{0}\right)=\log \left(\frac{1}{k_{0}}\right)-B,
$$

where we have used $\log \left(\frac{\Delta}{k}\right)-B=\log \left(\frac{1}{k}\right)+\log \Delta-B$ and redefined $B$ accordingly. See Figure 12 for a sketch of the "unrolled" geometry.

We have made no attempt to estimate errors in this derivation; indeed, we shall even be concerned with "large" values of $\varepsilon$ in $\$ 5.3$. Our derivation is in the spirit of that of the celebrated Hénon map [7]. However, note that the properties of $F$ are similar to those of $\mathcal{P}$ near $W^{\mathrm{u}}(\Gamma)$ and $W^{\mathrm{s}}(\Gamma): \triangle K(0, z)=\frac{6}{5} \varepsilon\left(z^{2}-a\right)+O\left(\varepsilon^{2}\right)$ corresponds to the shift $\varepsilon\left[A-\left(z_{0}+\varepsilon f\left(k_{0}\right)\right)^{2}\right]$ in (5.1) (we take $k \rightarrow-k$ in defining $F$ ), and the estimate (2.10) corresponds to the shift $\varepsilon\left[\log \left(\frac{1}{k_{0}}\right)-B\right]$ ( $B$ plays the rôle of $-b$ in (2.1)). Moreover, $T_{01}$ retains the behavior of the vector field described in Lemmas 3.1 and 3.2 of [2] and Lemma 2.1 above: orbits entering at a distance $k_{0}=\delta$ from $W^{\mathrm{s}}(\Gamma)$ leave at a distance $k_{1}=\delta$ from $W^{\mathbf{u}}(\Gamma)$, while being carried up $O(\varepsilon|\log \delta|)$ in $z$. Note however, that the $O\left(\varepsilon^{2}\right)$ term $\varepsilon f\left(k_{0}\right)$ term in $\left(z_{0}+\varepsilon f\left(k_{0}\right)\right)^{2}$ in (5.1) has no obvious quantitative equivalent in $\triangle K(0, z)$. This might give $F$ some special properties not possessed by $\mathcal{P}$; for example, as we see below, $F$ is area-preserving while $\mathcal{P}$ need not be so (and certainly is not for $c \neq 0$ in $(2.2-2.3)$, cf. Theorem 2.5 .

In the remainder of this section we study the dynamics of $F$; in particular, in Section 5.3 we prove that, for appropriate ranges of the parameters $A, B, \varepsilon>0, F$ has an invariant set topologically (semi-) conjugate to a full shift on two symbols: a Smale horseshoe (Ch. 5 of $[6])$. 

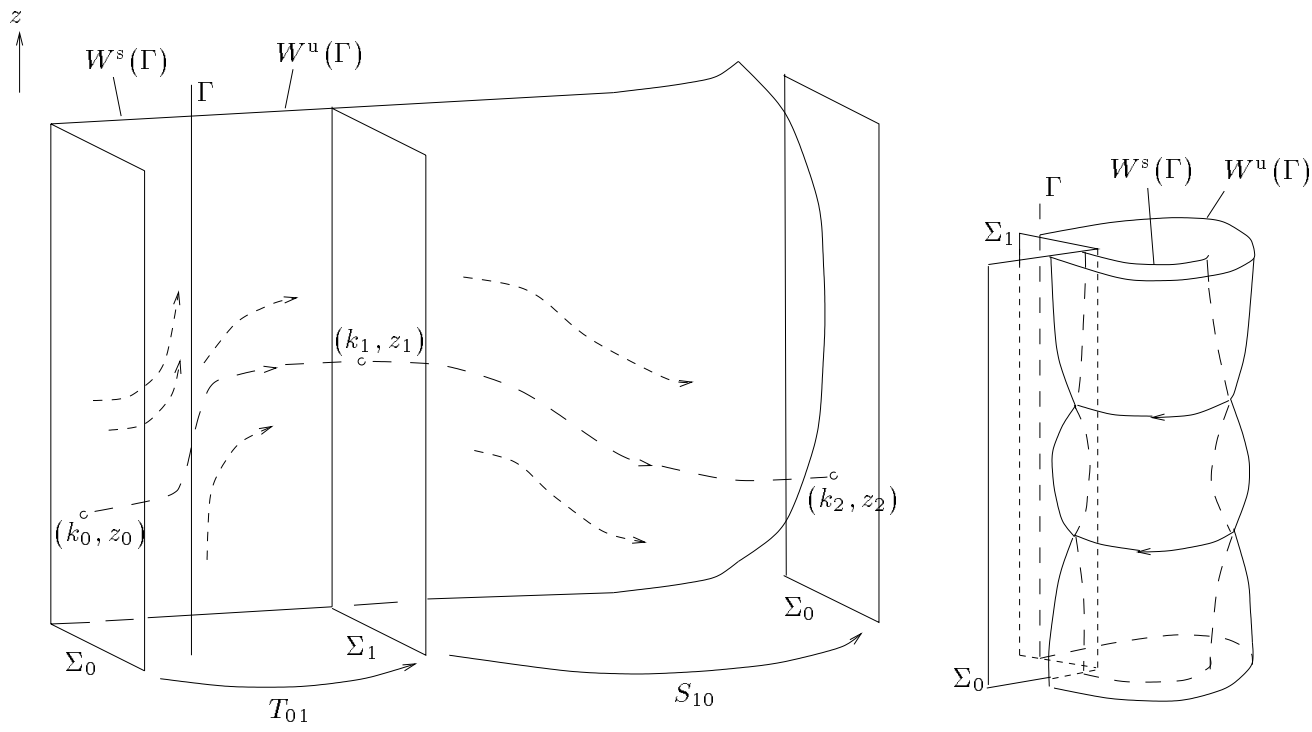

Figure 12: The geometry of the model map $F=S_{10} \circ T_{01}: \Sigma_{0} \rightarrow \Sigma_{0}$, unrolled. The right hand copy of $\Sigma_{0}$ can in essence be interpreted as a part of the cross section $\mathcal{V}=\{y=$ $0, x>1$ \} for the flow, viewed from behind.

First observe that, for $A>0, F$ has a pair of fixed points $(k, z)=\left(e^{-B}, \pm \sqrt{A}\right)$. The linearization

$$
D F(k, z)=\left(\begin{array}{cc}
1-2 \varepsilon^{2}(z+\varepsilon f(k)) f^{\prime}(k) & -2 \varepsilon(z+\varepsilon f(k)) \\
\varepsilon f^{\prime}(k) & 1
\end{array}\right)
$$

satisfies $\operatorname{det}(D F)=1, \operatorname{trace}(D F)=2\left[1-\varepsilon^{2}(z+\varepsilon f(k)) f^{\prime}(k)\right]$, so at the fixed points we have $f\left(e^{-B}\right)=0, f^{\prime}\left(e^{-B}\right)=-e^{B}$ and $\operatorname{trace}(D F)=2\left(1 \pm \varepsilon^{2} \sqrt{A} e^{B}\right)$. Hence $\left(e^{-B},+\sqrt{A}\right)$ is a hyperbolic saddle with positive eigenvalues for all $A>0, B>0$ and $\varepsilon>0$, while $\left(e^{-B},-\sqrt{A}\right)$ is a center for $A>0$ and $0<\varepsilon^{2} \sqrt{A} e^{B}<2$, and a hyperbolic saddle with negative eigenvalues for $\varepsilon^{2} \sqrt{A} e^{B}>2$. A period-doubling bifurcation occurs at $\varepsilon^{2} \sqrt{A} e^{B}=$ $2(§ 3.4$ of $[6])$; note that $B=-\mathcal{O}(|\log \varepsilon|)$ is necessary for this. These fixed points are analogues of the points $p$ and $q$ and the corresponding pair of periodic orbits found in the perturbation calculations of $\$ 2.3$.

\subsection{A topological horseshoe for small $\varepsilon$}

The horseshoe of the Poincaré map $\mathcal{P}$ has its origin in two mechanisms: (i) the exponential stretching and bending due to the fact that orbits close to $P(\Gamma)$ and $P^{-1}(\Gamma)$ are caught by the slow flow near $\Gamma$ and (ii) the averaged fast counteracting flow that balances this effect. Both mechanisms are preserved in the model mapping $F$, and assuming that $0<\varepsilon \ll 1$ and $B=O(|\log \varepsilon|)$, we can construct a topological horseshoe for $F$ very similar to that found in Section 3 for $\mathcal{P}$. Moreover, we can determine explicitly the analogues of the bifurcation curves $B_{2}\left(\cdot{ }_{2}^{1} 2\right), B_{3}\left(\cdot{ }_{2}^{1} \frac{1}{2} 2\right)$, etc. We introduce $\beta$ by

$$
B=\beta \log \frac{1}{\varepsilon}
$$




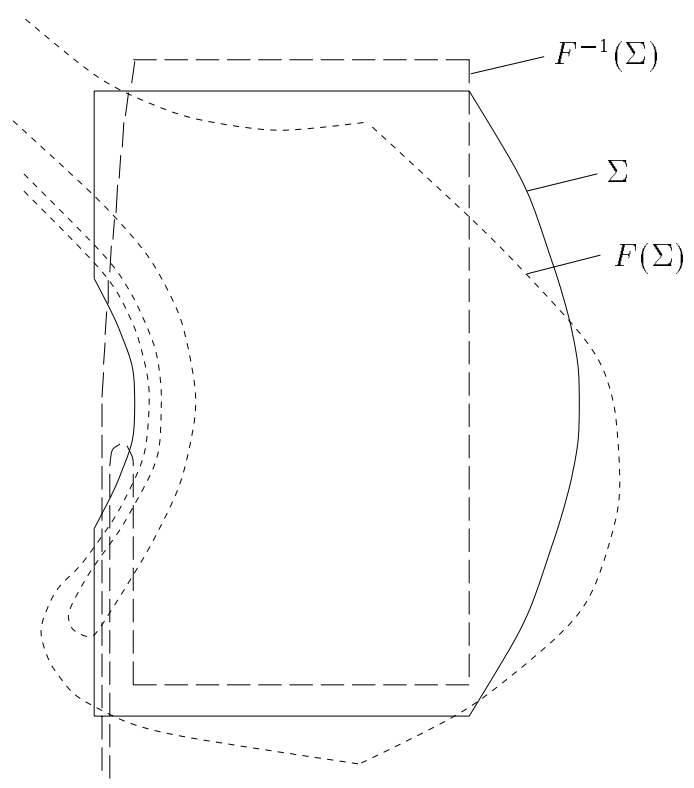

Figure 13: The regions $\Sigma, F(\Sigma)$ and $F^{-1}(\Sigma)$.

so the fixed points of $F$ are now $\left(\varepsilon^{\beta}, \pm \sqrt{A}\right)$. The counterpart $\Sigma \in(k, z)$-space of the region $S$ introduced in Section 3 (Figure 4) is determined by its boundary $\partial \Sigma=\sigma_{1} \cup \sigma_{2} \cup \ldots \cup \sigma_{6}$, where

$$
\begin{aligned}
& \sigma_{1}=\{k=0, z \in[\sqrt{A}, 2 \sqrt{A}]\} \\
& \sigma_{2}=\left\{k=\varepsilon\left(A-z^{2}\right), z \in[-\sqrt{A}, \sqrt{A}]\right\} \\
& \sigma_{3}=\{k=0, z \in[-\sqrt{A},-2 \sqrt{A}]\} \\
& \sigma_{4}=\{z=-2 \sqrt{A}, k \in[0,1-3 \varepsilon \sqrt{A}]\} \\
& \sigma_{5}=\left\{k=1+\varepsilon\left(A-z^{2}\right), z \in[-2 \sqrt{A}, 2 \sqrt{A}]\right\} \\
& \sigma_{6}=\{z=2 \sqrt{A}, k \in[0,1-3 \varepsilon \sqrt{A}]\} .
\end{aligned}
$$

See Figure 13. The exact choices of $\sigma_{4,5,6}$ are not crucial, but $\sigma_{1,2,3}$ are chosen such that the exponential stretching and bending occurs near this part of the boundary. Note that the inverse map $F^{-1}$ can be explicitly calculated:

$$
F^{-1}(k, z)=\left(k-\varepsilon\left[A-z^{2}\right], z-\varepsilon f\left[k-\varepsilon\left(A-z^{2}\right)\right]\right)
$$

with $f$ as in (5.1). Thus, the rôle of $P(\Gamma)$ (resp. $P^{-1}(\Gamma)$ ), i.e. the boundary of the region in which $\mathcal{P}^{-1}$ (resp. $\mathcal{P}$ ) is defined, is now played by $\left\{k=\varepsilon\left(A-z^{2}\right)\right\} \supset \sigma_{2}$ (resp. $\{k=0\} \supset \sigma_{1} \cup \sigma_{3}$ ), the boundary of the region where $F^{-1}$ (resp. $F$ ) is defined.

Tongue-like structures analogous to those of $P^{-k}(\Gamma)$ can be determined by calculating the images $F^{-1}\left(\sigma_{3}\right)$ and $F\left(\sigma_{2}\right)$. We first take points in $\sigma_{3}$ with $z$ coordinates $O(1)$ away from $-\sqrt{A}$ and find

$$
F^{-1}(0, z)=\left(\varepsilon\left(z^{2}-A\right), z-\varepsilon(1-\beta) \log \frac{1}{\varepsilon}\right)+\text { h.o.t. }
$$




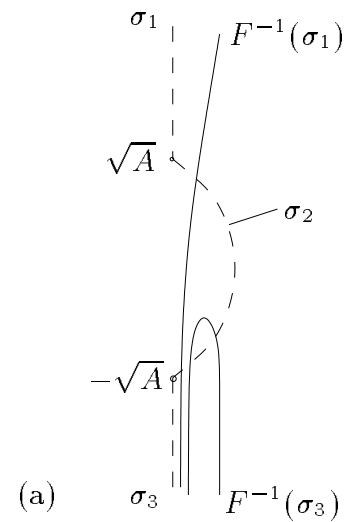

Figure 14: The curves $F^{-1}\left(\sigma_{3}\right)$ and $F^{-1}\left(\sigma_{1}\right)\left(\right.$ a) and $F\left(\sigma_{2}\right)$ (b)

thus, $F^{-1}$ shifts the $k$-coordinates (resp. $z$-coordinates) of these points $O(\varepsilon)$ to the right (resp. $O\left(\varepsilon \log \frac{1}{\varepsilon}\right)$ up or down, depending on $\beta$ ). Closer to the critical point $(0,-\sqrt{A})$ we set $z=-\sqrt{A}-\varepsilon^{\alpha} \zeta$, with $\alpha, \zeta>0$ and find

$$
F^{-1}(0, z)=\left(2 \varepsilon^{1+\alpha} \sqrt{A} \zeta,-\sqrt{A}-\varepsilon^{\alpha} \zeta-\varepsilon(1+\alpha-\beta) \log \frac{1}{\varepsilon}\right)+\text { h.o.t. }
$$

i.e. the shift in the $z$-coordinate increases as $\alpha$ increases. This mechanism is by construction exponentially strong: if we set $z=-\sqrt{A}-\zeta \exp \left(-\frac{1}{\varepsilon^{\alpha}}\right)$ (with $\alpha, \zeta>0$ ) we observe

$$
F^{-1}(0, z)=\left(\text { exp. small, }-\frac{1}{\varepsilon^{\alpha-1}}+\text { h.o.t. }\right) \text {. }
$$

The resulting (root) tongue $F^{-1}\left(\sigma_{3}\right)$ is shown in Figure 14(a), where we also show the branch $F^{-1}\left(\sigma_{1}\right)$ that runs 'parallel' to $k=0$. In Figure 14(b) we show the (unbounded) tongue $F\left(\sigma_{2}\right)$ that is folded along $\left\{k=\varepsilon\left(A-z^{2}\right)\right\}$. The calculations leading to $F^{-1}\left(\sigma_{1}\right)$ and $F\left(\sigma_{2}\right)$ are completely analogous to those above. Computations of $F^{ \pm}\left(\sigma_{4,5,6}\right)$ are even more straightforward; together with the above they lead to the picture of $F(\Sigma)$ and $F^{-1}(\Sigma)$ shown in Figure 13. We can now formulate the equivalent of Theorem 3.1:

Theorem 5.1 For $B>2 \log \frac{1}{\varepsilon}$ the map $F$ has an invariant set $\Lambda$ which is a topological horseshoe.

The fact that the critical value of $\beta$ is 2 (cf. 5.3) follows from (5.5): the $O\left(\varepsilon \log \frac{1}{\varepsilon}\right)$ term dominates the $z$-component of $F^{-1}(0, z)$ for $\alpha \geq 1$, the upward effect of $\beta$ can bring the tip of $F^{-1}\left(\sigma_{3}\right)$ above the line $\{z=-\sqrt{A}\}$ when $\beta>2$. However, it is not yet clear whether this is sufficient for a full topological horseshoe. As for $\mathcal{P}$ it is necessary to have an intersection of the $F^{-1}\left(\sigma_{3}\right)$-tongue with the $\sigma_{2}$ part of $\partial \Sigma$, or equivalently, an intersection of the $F\left(\sigma_{2}\right)$-tongue with the $\sigma_{3}$ part of $\partial \Sigma$ (see Figure 13). Inserting the general scaling

$$
z=-\sqrt{A}-\zeta \varepsilon^{\alpha_{1}}\left(\log \frac{1}{\varepsilon}\right)^{\alpha_{2}}, \alpha_{1}>0, \alpha_{2} \in \mathbf{R}, \zeta>0
$$

into $F^{-1}$ (with $k=0$ ), we find that the tip of the $F^{-1}\left(\sigma_{3}\right)$-tongue is described by values of $z$ with $\alpha_{1}=1$ and $\alpha_{2}=0$. Introducing $\beta_{1}$ by

$$
\beta=2+\beta_{1}\left(\log \frac{1}{\varepsilon}\right)^{-1} \text { or } B=2 \log \frac{1}{\varepsilon}+\beta_{1}
$$




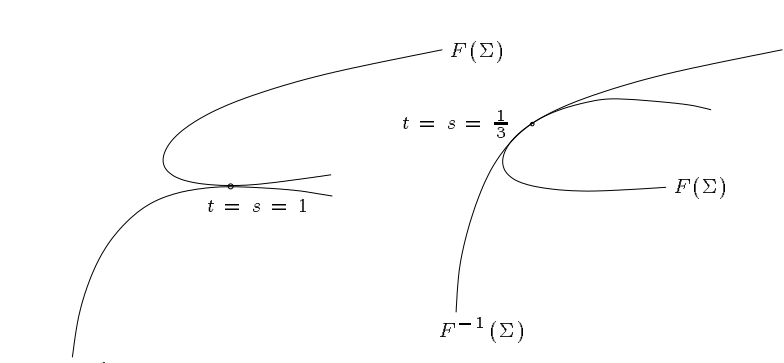

(a)

$F^{-1}(\Sigma)$

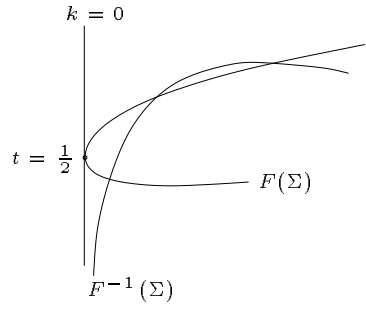

(c)

Figure 15: Three bifurcations: $B_{3}$ (first) (a) and $B_{3}$ (second) (b), and the bifurcation $B_{h s}$ (c).

(via (5.3)) and $\zeta>0$ by $z=-\sqrt{A}-\varepsilon \zeta$, yields that the tip of the $F^{-1}\left(\sigma_{3}\right)$-tongue is described at leading order by

$$
(k, z) \mapsto\left(2 \varepsilon^{2} \sqrt{A} \zeta,-\sqrt{A}-\varepsilon[\zeta-C-\log \zeta]\right),
$$

where $C=\beta_{1}+\log 2 \sqrt{A}$. Analogously the tip of the $F\left(\sigma_{2}\right)$-tongue is given at leading order by

$$
(k, z) \mapsto\left(2 \varepsilon^{2} \sqrt{A}[2 \xi-C-\log \xi],-\sqrt{A}+\varepsilon[\xi-C-\log \xi]\right)
$$

for $z=-\sqrt{A}+\varepsilon \xi$. Straightforward rescalings of $(k, z), \zeta, \xi$ into $(x, y), s, t$ and neglect of higher order terms then give the following scaled descriptions of the two critical 'tips':

$$
\begin{aligned}
F^{-1}\left(\sigma_{3}\right): & \{(s,-s+C+\log s), s \geq 0\} \\
F\left(\sigma_{2}\right): & \{(2 t-C-\log t, t-C-\log t), t \geq 0\} .
\end{aligned}
$$

Note that at the tips the a priori two-parameter family of maps $F$ has reduced to a one-parameter $(C)$ family at this order of approximation. Using the relatively simple expressions (5.6) we can explicitly determine the value of $B$ at which the $F$ gives has a full topological horseshoe (cf. Figure 15(c)):

$$
B_{h s}=B_{2}\left(\cdot \frac{1}{2} 2\right)=2 \log \frac{1}{\varepsilon}+1+\log 2-\log 2 \sqrt{A}+\text { h.o.t. }
$$

Thus, we can now sharpen the condition $B>2 \log \frac{1}{\varepsilon}$ to $B>B_{h s}$ in Theorem 5.1. We can also, for example, determine the two bifurcations $B_{3}$ (first) $<B_{3}$ (second) $<B_{h s}$ preceding this last horseshoe bifurcation. In Figure 15(b) we see that the tongue of $F^{-1}(\Sigma)$ and $F(\Sigma)$ have third order contact in the second bifurcation. Hence, at leading order a mixed scenario like the one sketched in Figure 10(c) occurs (in reverse order, since $B \sim-b$ ). In $B_{3}$ (first) the orbit $\cdot 112$ and a second 3 -pulse orbit arise, in $B_{3}$ (second) the latter orbit undergoes a pitchfork bifurcation to the orbits $\cdot 122, \cdot 212$ and $\cdot 222$. Thus we know explicitly which homoclinic bifurcations occur for $F$. The calculations give:

$$
\begin{aligned}
& B_{3}(\text { first }) \\
& B_{3}(\text { second })=2 \log \frac{1}{\varepsilon}+1-\log 2 \sqrt{A}+\text { h.o.t. } \\
& =2 \log \frac{1}{\varepsilon}+\frac{1}{3}+\log 3-\log 2 \sqrt{A}+\text { h.o.t. }
\end{aligned}
$$


Since neither $B_{3}\left(\cdot 2{ }_{2}^{1} 2\right)<B_{3}\left(\cdot 1_{2}^{1}\right.$ ) (where $F^{-1}(\Sigma)$ penetrates $F(\Sigma)$, Figure $10(\mathrm{a})$ ), nor $B_{3}\left(\cdot{ }_{2}^{1} 12\right)<B_{3}\left(\cdot{ }_{2}^{1} 22\right)$ (where $F(\Sigma)$ penetrates $F^{-1}(\Sigma)$, Figure $10(\mathrm{~b})$ ), is preferred by $F$ at leading order, either bifurcation sequence can generically occur. The one which does could be determined by higher order calculations, in which we would again have a 2-parameter problem (i.e. $A$ and $B$ (or $\beta_{1}$ ) instead of only $C$ ). Thus one expects a curve in the $A, B$ plane that distinguishes between the two generic orderings.

\subsection{Hyperbolicity of the horseshoe for larger $\varepsilon$}

For what follows, we will also require an expression for the derivative of $F^{-1}$, which may be found to be

$$
D F^{-1}(k, z)=\left(\begin{array}{cc}
1 & 2 \varepsilon z \\
-\varepsilon f^{\prime}\left(k-\varepsilon\left(A-z^{2}\right)\right) & 1-2 \varepsilon^{2} z f^{\prime}\left(k-\varepsilon\left(A-z^{2}\right)\right)
\end{array}\right) .
$$

We first construct a set $D$ analogous to $S$ of $\S 3.1$ and $\Sigma$ above, but simpler in shape, so that explicit estimates on the location of images and preimages can be calculated. This will lead to relatively crude estimates, which could probably be sharpened and brought closer to the bifurcation sets for topological horseshoes derived above.

Noting that $F$ maps vertical line segments $k=$ const. to arcs of parabolas, and arcs $z+\varepsilon f(k)=$ const. to horizontal line segments, we define $D$ as the simply-connected region enclosed by

$$
z+\varepsilon f(k)= \pm \kappa \sqrt{A} ; \quad k=e^{ \pm 2 \kappa \sqrt{A} / \varepsilon-B}
$$

for some $\kappa>1$. We claim that the parameters $A<B<\varepsilon$ and $\kappa$ can be chosen such that $D$ and $F(D)$ appear as in Figure 16, with $D \cap F(D)=V_{1} \cup V_{2}$ forming two disjoint "horizontal" strips and $F^{-1}(D) \cap D=H_{1} \cup H_{2}$ two disjoint "vertical" strips. In this figure the letters $a, b, \ldots, h$ denote points on $\partial D$ and $a^{\prime}, b^{\prime}, \ldots, h^{\prime}$ their images under $F$.

Lemma 5.2 For parameter values $A, B, \varepsilon>0$ and $\kappa>1$ such that

$$
e^{B}>\max \left\{\frac{2 \sinh (2 \kappa \sqrt{A} / \varepsilon)}{\varepsilon A\left(\kappa^{2}-1\right)}, \frac{2 \sinh (2 \kappa \sqrt{A} / \varepsilon)}{\varepsilon A}\right\}
$$

the point $d^{\prime}$ lies to the right of $k=e^{2 \kappa \sqrt{A} / \varepsilon-B}$ and $g^{\prime}$ lies to the left of $c$, so that $D \cap$ $F(D)$ forms two disjoint strips bounded vertically between $\left[z_{1}^{-}, z_{2}^{-}\right]$and $\left[z_{2}^{+}, z_{1}^{+}\right]$respectively, where

$$
\begin{aligned}
& z_{1}^{ \pm}= \pm \sqrt{A+\frac{2 e^{-B}}{\varepsilon} \sinh \left(\frac{2 \kappa \sqrt{A}}{\varepsilon}\right)}, \\
& z_{2}^{ \pm}= \pm \sqrt{A-\frac{2 e^{-B}}{\varepsilon} \sinh \left(\frac{2 \kappa \sqrt{A}}{\varepsilon}\right)} .
\end{aligned}
$$

Proof: This is a direct computation, using the locations and images of selected points as follows. Letting $C=e^{2 c \sqrt{A} / \varepsilon}$, we have

$$
\begin{array}{lll}
d=\left(\frac{e^{-B}}{C},-2 \kappa \sqrt{A}\right) & \stackrel{F}{\longmapsto} & \left(\frac{e^{-B}}{C}+\varepsilon A, 0\right)=d^{\prime}, \\
g=\left(C e^{-B}, \kappa \sqrt{A}\right) & \stackrel{F}{\mapsto} & \left(C e^{-B}-\varepsilon A\left(\kappa^{2}-1\right),-\kappa \sqrt{A}\right)=g^{\prime},
\end{array}
$$




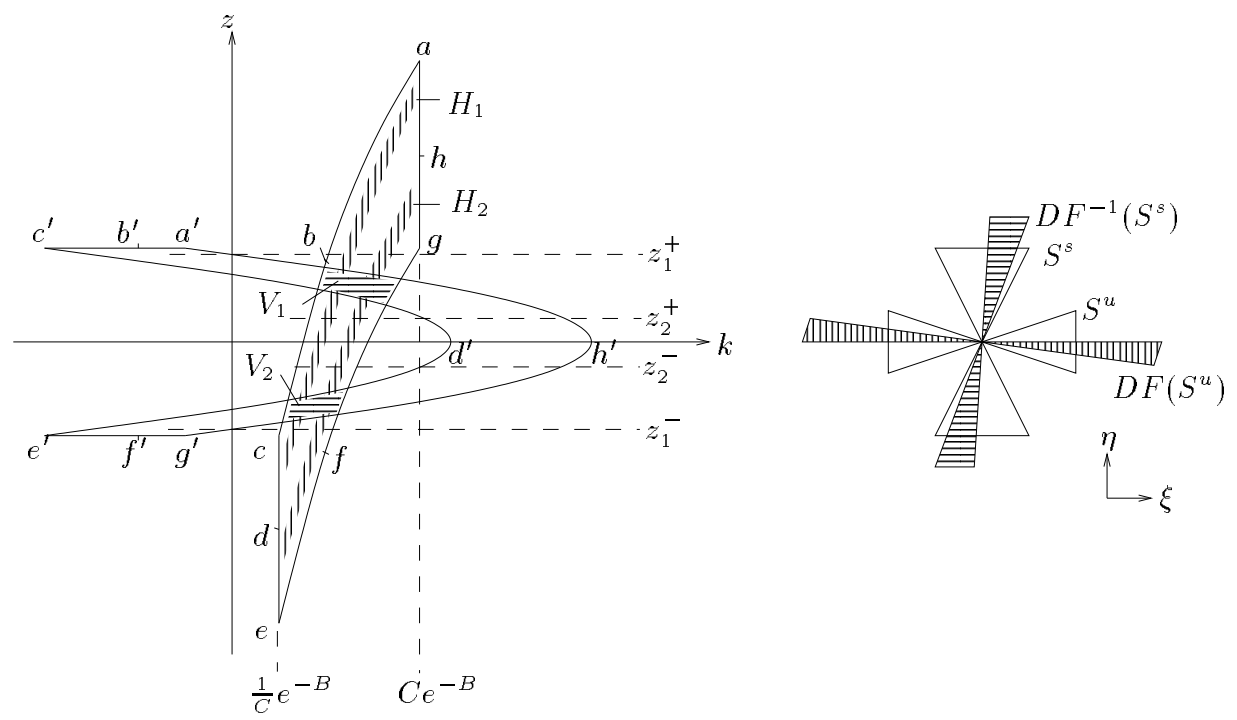

Figure 16: Bounds on strips, and stable and unstable cones.

and noting that $c=\left(\frac{e^{-B}}{C},-\kappa \sqrt{A}\right)$, the first assertion follows if

$$
e^{B}>\max \left\{\frac{C^{2}-1}{C \varepsilon A}, \frac{C^{2}-1}{C \varepsilon A\left(\kappa^{2}-1\right)}\right\},
$$

which, rewriting, gives (5.9).

For the (crude) bounds on the strips we compute the $z$ coordinates of appropriate intersections of the parabolas $a^{\prime} h^{\prime} g^{\prime}$ and $c^{\prime} d^{\prime} e^{\prime}$ given by

$$
k=C e^{-B}+\varepsilon\left(A-z^{2}\right), k=\frac{e^{-B}}{C}+\varepsilon\left(A-z^{2}\right)
$$

with their preimages, the vertical lines $a h g$ and $c d e$ :

$$
k=C e^{-B}, k=\frac{e^{-B}}{C} .
$$

We note that (5.9) implies that the second square root in (5.10) is well defined. Also observe that these latter bounds imply that the strips $F^{-1}(D) \cap D$ lie between the curves

$$
z+\varepsilon f(k)=z_{1}^{-}, z_{2}^{-} \text {and } z+\varepsilon f(k)=z_{1}^{+}, z_{2}^{+}
$$

respectively, and that the fixed points $\left(e^{-B}, \pm \sqrt{A}\right)$ lie in $F^{-1}(D) \cap D \cap F(D)$.

This lemma implies that the invariant set $\Lambda=\bigcap_{n=-\infty}^{\infty} F^{n}(D)$ is a topological horseshoe. To prove hyperbolicity, we must verify that the linearized map $D F$ and its inverse $D F^{-1}$ additionally satisfy the condition $i i i$ of Moser [15], pages 76-77 (c.f. [6], hypothesis $H 3$ and Theorem 5.2.4, pp. 240-241). Noting that $F$ is an area-preserving map, we have to find stable and unstable cones

$$
S^{s}=\{(\xi, \eta):|\xi|<\mu|\eta|\}, \quad S^{u}=\{(\xi, \eta):|\eta|<\mu|\xi|\},
$$


for some $0<\mu<\frac{1}{2}$, such that $D F\left(S^{u}\right) \subset S^{u}$ and $D F^{-1}\left(S^{s}\right) \subset S^{s}$ and, if $D F\left(\xi_{0}, \eta_{0}\right)=$ $\left(\xi_{1}, \eta_{1}\right)$ and $D F^{-1}\left(\xi_{0}, \eta_{0}\right)=\left(\xi_{-1}, \eta_{-1}\right)$ then $\left|\xi_{1}\right| \geq \frac{1}{\mu}\left|\xi_{0}\right|$ and $\left|\eta_{-1}\right| \geq \frac{1}{\mu}\left|\eta_{0}\right|$. (Note the reversal of the roles of $\xi$ and $\eta$, compared with [6].) See Figure 16.

Using the expression (5.2) for $D F$, we see that $D F\left(S^{u}\right) \subset S^{u}$ provided that the vector $\left(1, \eta_{0}\right)^{T}$ has image $\left(\xi_{1}, \eta_{1}\right)^{T}$ with

$$
\left|\frac{\eta_{1}}{\xi_{1}}\right|=\left|\frac{\varepsilon f^{\prime}(k)+\eta_{0}}{1-2 \varepsilon(z+\varepsilon f(k))\left(\varepsilon f^{\prime}(k)+\eta_{0}\right)}\right| \leq \mu
$$

or, rewritten,

$$
\left|2 \varepsilon(z+\varepsilon f(k))+\frac{1}{\left(\varepsilon / k-\eta_{0}\right)}\right| \geq \frac{1}{\mu}, \quad \forall\left|\eta_{0}\right| \leq \mu,
$$

where we use $f^{\prime}(k)=-\frac{1}{k}$. From the trace $(D F)$ calculations above, we note that $z+\varepsilon f(k)$ must be large in magnitude for the lower fixed point to be a hyperbolic saddle; clearly a necessary condition for hyperbolicity of $\Lambda$. The proof of Lemma 5.2 and (5.8) imply that satisfying (5.11) for $z+\varepsilon f(k) \in\left[z_{1}^{-}, z_{2}^{-}\right] \cup\left[z_{2}^{+}, z_{1}^{+}\right]$and $k \in\left[e^{-2 \kappa \sqrt{A} / \varepsilon-B}, e^{2 \kappa \sqrt{A} / \varepsilon-B}\right]$ is sufficient. (Note that $z=z_{2}^{ \pm}$give worse cases than $z=z_{1}^{ \pm}$.)

An analogous calculation using (5.7) shows that $D F^{-1}\left(S^{s}\right) \subset S^{s}$ provided that

$$
\left|\frac{\varepsilon}{k-\varepsilon\left(A-z^{2}\right)}+\frac{1}{\xi_{0}+2 \varepsilon z}\right| \geq \frac{1}{\mu}, \quad \forall\left|\xi_{0}\right|<\mu,
$$

where in turn it is sufficient to satisfy the inequality for the worst cases $z=z_{2}^{ \pm}$and $k-\varepsilon\left(A-z^{2}\right) \in\left[e^{-2 \kappa \sqrt{A} / \varepsilon-B}, e^{2 \kappa \sqrt{A} / \varepsilon-B}\right]$.

To check the conditions on expansion of $\xi_{1}$ components under $D F$ we compute

$$
\left|\xi_{1}\right|=\left|1-2 \varepsilon(z+\varepsilon f(k))\left(\varepsilon f^{\prime}(k)+\frac{\eta_{0}}{\xi_{0}}\right)\right| \cdot\left|\xi_{0}\right|=\left|1+2 \varepsilon(z+\varepsilon f(k))\left(\frac{\varepsilon}{k}-\frac{\eta_{0}}{\xi_{0}}\right)\right| \cdot\left|\xi_{0}\right|,
$$

so that the condition is satisfied provided

$$
\left|1+2 \varepsilon(z+\varepsilon f(k))\left(\frac{\varepsilon}{k}-\frac{\eta_{0}}{\xi_{0}}\right)\right| \geq \frac{1}{\mu}, \quad \forall\left|\frac{\eta_{0}}{\xi_{0}}\right| \leq \mu .
$$

Again the worst cases are given by $z+\varepsilon f(k)=z_{2}^{ \pm}$and $k=e^{ \pm 2 \kappa \sqrt{A} / \varepsilon-B}$. An analogous condition implies expansion of $\eta_{-1}$ components under $D F^{-1}$ :

$$
\left|\eta_{-1}\right|=\left|-\varepsilon f^{\prime}\left(k-\varepsilon\left(A-z^{2}\right)\right)\left(\frac{\xi_{0}}{\eta_{0}}\right)+1-2 \varepsilon^{2} z f^{\prime}\left(k-\varepsilon\left(A-z^{2}\right)\right)\right| \cdot\left|\eta_{0}\right| \geq \frac{1}{\mu}\left|\eta_{0}\right|
$$

or, rewritten,

$$
\left|1+\frac{\varepsilon\left(2 \varepsilon z+\left(\frac{\xi_{0}}{\eta_{0}}\right)\right)}{k-\varepsilon\left(A-z^{2}\right)}\right| \geq \frac{1}{\mu}, \quad \forall\left|\frac{\xi_{0}}{\eta_{0}}\right| \leq \mu .
$$

Here the worst case is given by $k-\varepsilon\left(A-z^{2}\right)=e^{ \pm 2 \kappa \sqrt{A} / \varepsilon-B}, z=z_{2}^{ \pm}$. We have proved 
Theorem 5.3 For parameter values $A, B, \varepsilon>0, \kappa>1$ such that the inequalities (5.9), (5.11), (5.12), (5.13) and (5.14) are satisfied for all $(k, z) \in D \cap F(D), \Lambda=\bigcap_{n=-\infty}^{\infty} F^{n}(D)$ is an invariant, hyperbolic Cantor set for $F$ (a Smale horseshoe). If (5.9) holds but one or more of the other inequalities fails, then $\Lambda$ is a topological Cantor set which may not be hyperbolic.

Remark 5.4 We can find open sets of parameter values satisfying these sufficient conditions. Without loss of generality we set $A=1$. Noting that $B=O(|\log \varepsilon|)$ is necessary for (5.9), but that $\sinh (2 \kappa \sqrt{A} / \varepsilon)=O\left(e^{2 \kappa \sqrt{A} / \varepsilon}\right)$ cannot be too large either, we pick $B$ and $\varepsilon$ both of $O(1)$. Specifically, setting $A=1, B=2, \varepsilon=3, \kappa=1.1$ all the inequalities are comfortably satisfied with $\mu=\frac{1}{2}$. However, since the left hand side of (5.11-5.14) is at most $\sqrt{A}=1$, it does not seem possible to satisfy the inequalities for arbitrarily small $\varepsilon$. If $\varepsilon$ is taken smaller, one must increase $A$ or $B$; for instance with $A=5, B=8$, $\varepsilon=1$ and $\kappa=1.1$ all inequalities are satisfied also. It would be interesting to study this systematically for decreasing $\varepsilon$ using computational tools such as [13].

\section{Conclusions and discussion}

In this paper we have significantly extended the analysis of a three-dimensional vector field begun in [2], which in turn built on the methods of [3]. The basic idea is to use geometric singular perturbation methods (Fenichel theory) to establish the quasi-local structure of certain stable and unstable manifolds to a slow manifold $\Gamma$. Melnikov type calculations enable us to approximate a Poincaré return map $\mathcal{P}$ and prove that quadratic tangencies and transverse intersections occur. We focus on the case in which the slow flow in the neighborhood of $\Gamma$ can be balanced by the counteracting effects of the fast flow, and a complicated non-wandering set $\Lambda$ - a Smale horseshoe - appears. The perturbation methods do not seem sufficient to give hyperbolicity of $\Lambda$, but the topological semi-conjugacy between orbits in $\Lambda$ and bi-infinite symbol sequences extends naturally to a symbolic description of the countable sets of homoclinic orbits to $\Gamma$. This enables us to conveniently describe and partially order homoclinic bifurcation curves in the $(a, b)$ parameter plane. This analysis occupies the bulk of the paper: Sections 2-4. A key idea is that, since the unstable and stable manifolds of $\Gamma$ intersect the stable and unstable manifolds of $\Lambda$, one can find orbits homoclinic to $\Gamma$ which remain under the influence of the chaotic dynamics of $\Lambda$ for as long as one wishes (Theorem 3.8).

To further illustrate our results, in Section 5 we formally derive an explicit return mapping $F$ which shares the key features of $\mathcal{P}$, and for which we can explicitly approximate some of the homoclinic bifurcation curves and, relaxing the small parameter condition, prove hyperbolicity via the sector bundle estimates of Moser. This exponential-quadratic map may be of general interest as an example of a singular mapping with complicated dynamics.

The major problem not addressed in this paper is the question of hyperbolicity of $\Lambda$ for small $\varepsilon$, which we were unable to prove even for the explicit map $F$ constructed in Section 5. The main obstruction seems to be the global interplay between the singular part of the map (near $P^{ \pm}(\Gamma)$ for $\mathcal{P}$ or $\sigma_{1} \cup \sigma_{2} \cup \sigma_{3}$ for $F$ ) and the counteracting regular part (the averaged fast flow). Such a structure may not be amenable to the uniform cone estimates of Moser's method. (We note that the horseshoes constructed in [12] have a 
more "local" character; there the key ingredient in proving hyperbolicity is the hyperbolic structure near the slow manifold.) Other open problems include the possibility that some of the $\Lambda^{N}$ 's defined in $\S 4.2$ may be hyperbolic even if $\Lambda$ is not. It would also be nice to obtain further explicit bifurcation orderings of the type given in $\$ 4.1$.

Although this class of problems was motivated by specific applications to BVPs arising in the search for traveling wave solutions of Ginsburg-Landau type PDEs, the methods are more generally applicable to near-integrable $n \geq 3$-dimensional flows.

Acknowledgments This work was supported by DoE Grant DE-FG02-95ER.25238.A000 (PH) and the Netherlands Organization for Scientific Research (AD). Erin Lynch's software for studying invariant domains and hyperbolicity of two-dimensional mappings [13] was particularly helpful in our construction and verification of the estimates in $§ 5.3$.

\section{References}

[1] A. Doelman. Breaking the hidden symmetry in the Ginzburg-Landau equation. Physica $D, 97: 398-428,1996$.

[2] A. Doelman and G. Hek. Homoclinic saddle-node bifurcations in singularly perturbed systems. submitted, 1997.

[3] A. Doelman and P. Holmes. Homoclinic explosions and implosions. Phil. Trans. R. Soc. Lond. A , 354:845-893, 1995.

[4] N. Fenichel. Geometric singular perturbation theory for ordinary differential equations. J. Diff. Eq., 31:53-98, 1979.

[5] R.W. Ghrist, P.J. Holmes, and M.C. Sullivan. Knots and Links in Three-Dimensional Flows. Springer Verlag, New York etc., 1997. Lecture Notes in Mathematics No. 1654.

[6] J. Guckenheimer and P.J. Holmes. Nonlinear oscillations, Dynamical systems and Bifurcations of Vector Fields. SpringerVerlag, New York etc., 1983.

[7] M. Hénon. A two-dimensional mapping with a strange attractor. Comm. Mth. Phys., 50, 1976.

[8] P. Holmes. Knotted periodic orbits in suspensions of Smale's horseshoe: extended families and bifurcation sequences. Physica D, 40:42-64, 1989.

[9] P. Holmes and D. Whitley. Bifurcations of one- and two-dimensional maps. Phil. Trans. R. Soc. Lond. A , 311:43-102, 1984.

[10] L.N. Howard and N. Kopell. Slowly varying waves and shock structures in reactiondiffusion equations. Studies in Appl. Math., 56:95-145, 1977.

[11] C.K.R.T. Jones, T.J. Kaper, and N. Kopell. Tracking Invariant Manifolds up to Exponentially Small Errors. SIAM J. Math. Anal., 27(2):558-577, 1996. 
[12] N. Kopell and L.N. Howard. Target patterns and horseshoes from a perturbed centralforce problem: some temporally periodic solutions to reaction-diffusion equations. Studies in Appl. Math., 64:1-56, 1981.

[13] E. Lynch. Hypmap: a java program. Available from World Wide Web site http://www.cds.caltech.edu/ elynch, 1998.

[14] W. van Saarloos and P. C. Hohenberg. Fronts, pulses, sources and sinks in generalized complex Ginzburg-Landau equations. Physica D, 56:303-367, 1992.

[15] J. Moser. Stable and Random Motions in Dynamical Systems. Princeton University Press, 1973.

[16] C. Robinson. Sustained resonance for a nonlinear system with slowly varying coefficients. SIAM Math. An., 14:847-860, 1983.

[17] L.P. Silnikov. A case of the existence of a denumerable set of periodic motions. Sov. Math. Dokl., 6:163-166, 1965. 